\title{
Epithelial membrane protein 2 governs transepithelial migration of neutrophils into the airspace
}

\author{
Wan-Chi Lin, ${ }^{1}$ Kymberly M. Gowdy, ${ }^{1}$ Jennifer H. Madenspacher, ${ }^{1}$ Rachel L. Zemans, ${ }^{2}$ Kazuko Yamamoto, ${ }^{3,4,5}$ \\ Miranda Lyons-Cohen, ${ }^{1}$ Hideki Nakano, ${ }^{1}$ Kyathanahalli Janardhan, ${ }^{6,7}$ Carmen J. Williams, ${ }^{8}$ Donald N. Cook, Joseph P. Mizgerd, ${ }^{3}$ \\ and Michael B. Fessler ${ }^{1}$ \\ IImmunity, Inflammation and Disease Laboratory, National Institute of Environmental Health Sciences, Research Triangle Park, North Carolina, USA. ²Department of Medicine, University of Michigan, Ann \\ Arbor, Michigan, USA. ${ }^{3}$ Pulmonary Center, Boston University School of Medicine, Boston, Massachusetts, USA. ${ }^{4}$ Second Department of Internal Medicine, Nagasaki University Hospital, Nagasaki, Japan. \\ ${ }^{5}$ Department of Clinical Research Center, National Hospital Organization Nagasaki Medical Center, Omura, Japan. ${ }^{6}$ Cellular \& Molecular Pathology Branch, National Toxicology Program, National Institute \\ of Environmental Health Sciences, Research Triangle Park, North Carolina, USA. IIntegrated Laboratory Systems Inc., Research Triangle Park, North Carolina, USA. ${ }^{8}$ Reproductive and Developmental Biology \\ Laboratory, National Institute of Environmental Health Sciences, Research Triangle Park, North Carolina, USA.
}

\begin{abstract}
Whether respiratory epithelial cells regulate the final transit of extravasated neutrophils into the inflamed airspace or are a passive barrier is poorly understood. Alveolar epithelial type 1 (AT1) cells, best known for solute transport and gas exchange, have few established immune roles. Epithelial membrane protein 2 (EMP2), a tetraspan protein that promotes recruitment of integrins to lipid rafts, is highly expressed in AT1 cells but has no known function in lung biology. Here, we show that Emp2 ${ }^{-/-}$ mice exhibit reduced neutrophil influx into the airspace after a wide range of inhaled exposures. During bacterial pneumonia, Emp2-/- mice had attenuated neutrophilic lung injury and improved survival. Bone marrow chimeras, intravital neutrophil labeling, and in vitro assays suggested that defective transepithelial migration of neutrophils into the alveolar lumen occurs in Emp2 $2^{-/-}$lungs. Emp2 ${ }^{-/-}$AT1 cells had dysregulated surface display of multiple adhesion molecules, associated with reduced raft abundance. Epithelial raft abundance was dependent upon putative cholesterol-binding motifs in EMP2, whereas EMP2 supported adhesion molecule display and neutrophil transmigration through suppression of caveolins. Taken together, we propose that EMP2-dependent membrane organization ensures proper display on AT1 cells of a suite of proteins required to instruct paracellular neutrophil traffic into the alveolus.
\end{abstract}

\section{Introduction}

Successful migration of neutrophils (PMNs) from the circulation to the airway lumen is critical for pulmonary host defense, but it is also responsible for bystander tissue injury that underlies a wide array of acute and chronic lung diseases. PMN trafficking to the lung differs fundamentally from that in other tissues (1). Integrin-dependent firm adhesion to endothelium and diapedesis occur in alveolar capillaries, after which PMNs are thought to track along interstitial fibroblasts, finally passing paracellularly through the epithelium at the junction of alveolar epithelial type 1 (AT1) and type 2 (AT2) cells (2). Studies over the past 2 decades have loosely categorized the PMN influx to different airway exposures on the basis of the PMN integrins utilized. Thus, trafficking in response to lipopolysaccharide (LPS), E. coli, and $P$. aeruginosa is primarily CD18-dependent ( $\beta 2$ integrin-dependent), whereas trafficking in response to $S$. pneumoniae and CXCL1 is CD18independent and CD29-dependent ( $\beta 1$ integrin-dependent) (3). Endothelial ligands that interact with PMN integrins to control vascular egress have been well described (3). Epithelial mem-

Authorship note: WCL and KMC contributed equally.

Conflict of interest: The authors have declared that no conflict of interest exists

Copyright: ๑ 2020, American Society for Clinical Investigation.

Submitted: January 2, 2019; Accepted: September 18, 2019; Published: November 18, 2019.

Reference information: J Clin Invest. 2020;130(1):157-170.

https://doi.org/10.1172/JCI127144. brane proteins also regulate transepithelial migration (TEM) via cognate interactions with PMNs (4), and studies suggest that transepithelial rather than transendothelial passage of PMNs is linked to epithelial wounding, catastrophic lung damage, and mortality $(4,5)$. Targeting pulmonary TEM might conceivably offer effective and selective strategies for PMN-mediated lung disease. However, to date, the few epithelial membrane proteins that have been proposed to regulate pulmonary TEM (e.g., intercellular adhesion molecule 1 [ICAM-1]; CD47) have largely been extrapolated from in vitro studies of intestinal epithelium $(4,6)$. AT1 cells are best known for their roles in solute transport and gas exchange. Whether AT1 cells regulate PMN TEM is unknown. Whether TEM-regulatory proteins are coordinated in alveolar epithelial cells is also an open question.

Epithelial membrane protein 2 (EMP2) is a member of the tetraspan superfamily of membrane proteins. Although its mechanism of action remains somewhat obscure, EMP2 is thought to promote the recruitment of select integrins ( $\alpha 6 \beta 1, \alpha v \beta 3)$, adhesion molecules (ICAM-1), and signaling proteins to plasma membrane raft microdomains, and to downregulate caveolins, thereby reciprocally augmenting rafts and reducing caveolae (7-10). In cancer cells, EMP2 may serve as a platform for integrin signaling, supporting cell adhesion to extracellular matrix (ECM) and other cytoskeletal functions (11). Of interest, in rodents and humans, EMP2 is by far most highly expressed in the lung (biogps.org), whereas EMP2 protein has been shown to be highly expressed in 
AT1 cells, but absent in AT2 cells and alveolar macrophages (AMs) (12). To date, however, no function has been assigned to EMP2 in lung biology, and few roles have been identified for AT1 cells in regulation of immune responses.

Here, we show that $E m p 2^{-/-}$mice exhibit deficient alveolar PMN influx in response to a wide range of CD18-dependent and -independent inhaled exposures. We provide evidence that this is due to deficient PMN TEM, arising from a deficit in epithelial function. Emp $2^{-/-}$AT1 cells are deficient in lipid rafts and surface adhesion molecules and Emp2-deficient epithelial cells support reduced PMN TEM in vitro. Mutagenesis of EMP2 reveals that its functions are dependent upon 2 putative cholesterol-binding sequences, suggesting that EMP2 serves a critical raft scaffolding function. EMP2 support of adhesion molecule display and PMN TEM is dependent upon epithelial cell caveolin-2. Emp $2^{-/-}$mice have reduced lung injury and enhanced survival during bacterial pneumonia. We identify EMP2 as a master regulator of AT1 membrane organization that instructs the terminal step of PMN traffic into the alveolus.

\section{Results}

EMP2 regulates TEM of PMNs into the alveolar space in response to diverse exposures. EMP2, a tetraspan membrane protein, is reportedly expressed in AT1, but not in AT2 cells or in AMs in rat and human lung (12). We found that Emp2 transcripts were readily detected in AT1 (CD45 $\left.{ }^{-} \mathrm{CD} 31^{-} \mathrm{CD} 34^{-} \mathrm{EpCAM}^{\text {int }} \mathrm{T} 1 \alpha^{+} \mathrm{MHCII}^{-}\right)$, AT2 $\left(\mathrm{CD} 45^{-} \mathrm{CD} 31^{-} \mathrm{CD} 34^{-} \mathrm{EpCAM}^{\mathrm{int}} \mathrm{T} 1 \alpha^{-} \mathrm{MHCII}^{+}\right)$, and airway epithelial cells $\left(\mathrm{CD} 45^{-} \mathrm{CD} 31^{-} \mathrm{CD} 34^{-} \mathrm{EpCAM}^{\mathrm{hi}} \mathrm{MHCII}^{-}\right)$sorted from murine lung, albeit with relative enrichment in the former cell type (Supplemental Figure 1; supplemental material available online with this article; https://doi.org/10.1172/JCI127144DS1). The specific signal for EMP2 protein was, however, observed only in AT1 cells (Supplemental Figure 2), suggesting posttranscriptional regulation. In vivo, Emp2 RNA and protein were both transiently downregulated in mouse lung following LPS inhalation, with a progressive return toward baseline commencing after 24 hours after exposure (Supplemental Figure 3, A and B). EMP2 was induced in a time-dependent fashion in both mouse and rat primary AT2 cells during in vitro transdifferentiation into AT1-like cells (Supplemental Figure 3, C and D). Surveying a panel of pulmonary epithelial cell lines, we found, somewhat surprisingly, that EMP2 protein was expressed in several airway lines (Calu-3, Beas-2B, H292) (Supplemental Figure 3E), but was undetectable in 2 AT1like cell lines (E10, Let1) (not shown).

Given that EMP2 supports lipid raft assembly $(8,9)$ and rafts regulate Toll-like Receptor (TLR) signaling (13), we hypothesized that EMP2 might be required for the pulmonary innate immune response. To test this, we studied Emp2-null mice (14). Naive 8- to 12-week-old Emp2 $2^{-/-}$mice had no overt changes in lung histology (not depicted), nor changes in resident AM number or bronchoalveolar lavage fluid (BALF) concentration of total protein or cytokines (Supplemental Figure $4 \mathrm{~A}-\mathrm{C}$ ), suggesting that EMP2 does not play a critical role in lung development. Pulmonary expression of the alternate tetraspan family members Emp1, Emp3, and Pmp22 was also unaltered (Supplemental Figure 4D). Immunoblotting revealed a modest reduction in the tight junction protein occludin in lung homogenates of naive Emp $2^{-/-}$mice, but no discernible change in expression of JAM-1 or claudins-3, -12, or -18 (Supplemental Figure 4E). Upon inhalational challenge, Emp $2^{-/-}$mice exhibited a striking reduction in alveolar neutrophilia triggered by LPS, Gram-negative bacteria (K. pneumoniae, P. aeruginosa), and Gram-positive bacteria (S. pneumoniae) (Figure 1, A-D), suggesting a wide-ranging deficit in innate immunity. Pretreatment of $E m p 2^{+/+}$mice i.t. with an anti-EMP2 scFv antibody fragment reported to inhibit EMP2 (15) also reduced LPS-induced airspace neutrophilia compared with control treatment (Supplemental Figure 5). Given that TLR activation in AMs and alveolar epithelial cells induces cytokines and chemokines that then attract circulating PMNs, we initially predicted that $E m p 2^{-/-}$mice would have a reduction in these mediators in the airspace. However, BALF concentrations of cytokines and chemokines of established importance to PMN lung trafficking (3) were all normal 2 hours after LPS inhalation, a time point prior to substantial PMN influx (Figure 1, A and E). Emp2 $2^{+/+}$and Emp2 $2^{-/-}$mice also had equivalent numbers of circulating PMNs in the naive, LPS-exposed, and K. pneumoniaeexposed state (Supplemental Figure 6), suggesting no defect in supply of peripheral PMNs. Collectively, this suggested to us either a cell-intrinsic or -extrinsic deficit in migration of circulating PMNs to the airspace. Given that Emp2 transcripts are minimally detectable in murine PMNs ( $>10$-fold lower in PMNs than in AMs by RNA-Seq [Immgen.org]), we hypothesized that a cellextrinsic mechanism was more likely.

In support of deficient PMN trafficking in Emp2 $2^{-/-}$mice, reduced influx of PMNs to the airspace was also observed after intrapulmonary inoculation of naive mice with the chemokine CXCL1 (Figure 1F). No differential secondary induction of CXCL5, reportedly produced by AT1 cells under some conditions (16), was observed in the airspace of CXCL1-inoculated $E m p 2^{-/-}$mice, suggesting that the differential PMN trafficking was a primary response to intraalveolar CXCL1. Notably, intraperitoneal injection of CXCL1 elicited equivalent PMN influx into the peritoneal cavities of $E m p 2^{+/+}$and $E m p 2^{-/-}$mice, formally demonstrating intact intrinsic chemotactic function of Emp2-/PMNs in vivo (Figure 1G).

Chimeric mice made by bone marrow transfer revealed that EMP2 deletion in radioresistant lung cells, but not radiosensitive (i.e., hematopoietic) cells was sufficient to confer deficient alveolar PMN influx in response to inhaled LPS (Figure 2A). This finding, in conjunction with the i.t. CXCL1 results and expression pattern of EMP2 in the murine lung, suggested that a defect in Emp $2^{-/-}$AT1 cells might be causing deficient TEM of PMNs from the pulmonary interstitium into the alveolar lumen. Supporting this idea, we found an approximately 2-fold increase in PMNs in the parenchyma of perfused Emp2 $2^{-1-}$ lungs after LPS challenge (Figure 2B). Intravital PMN labeling with i.v. antibody to distinguish endovascular from interstitial (extravascular) PMNs in perfused and airwaylavaged lungs confirmed a significant increase in interstitial PMNs in Emp $2^{-/-}$mice following LPS (Figure 2C), pointing to a deficit in PMN transit from interstitium to airspace lumen. This was further corroborated by live lung slice imaging, which interestingly revealed a marked increase in interstitial PMNs both in the bronchovascular sheath surrounding, and in the wall of, small airways (Figure 2D). This result raises the possibility of an epithelial abnormality at the level of small airways in $E m p 2^{-/-}$mice. Given that 
A

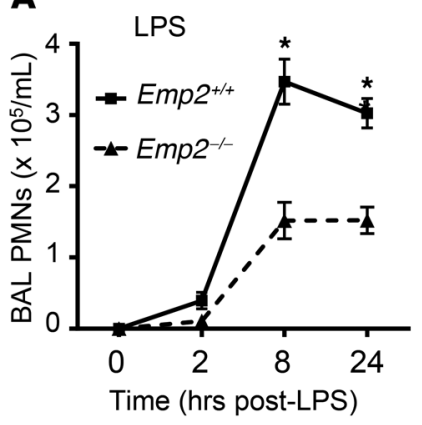

E

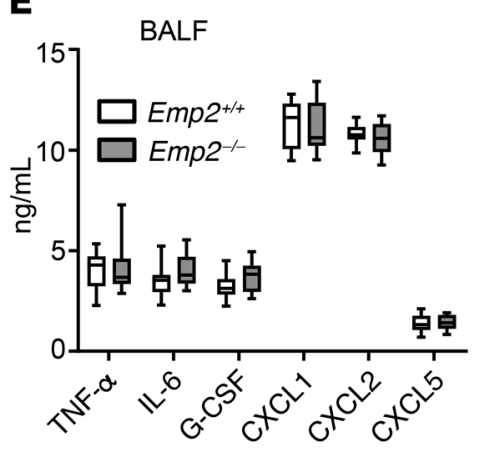

B

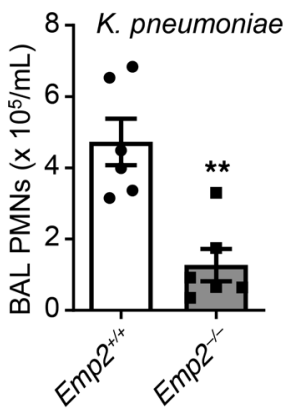

$\mathbf{F}$

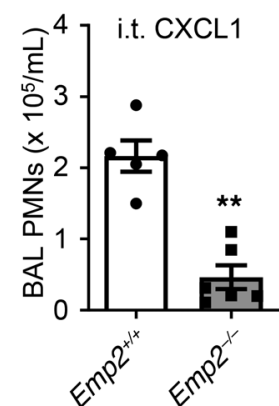

C
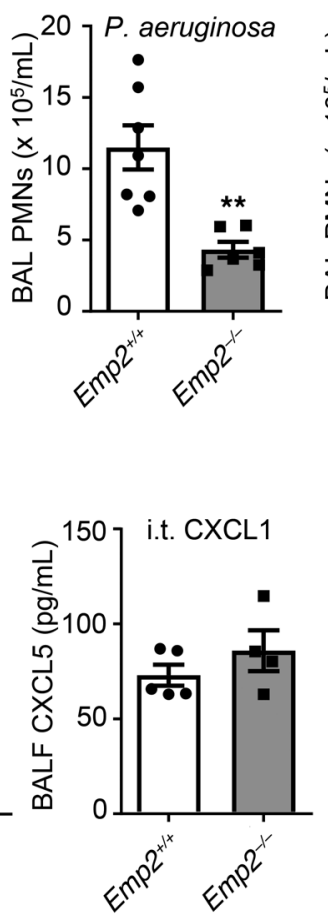

D

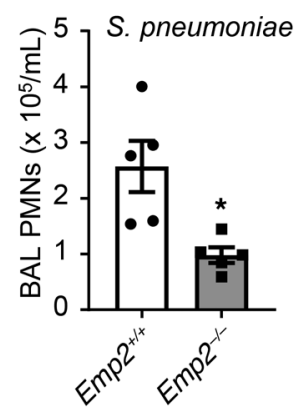

G

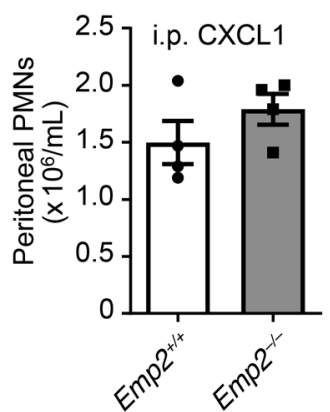

Figure 1. EMP2 regulates trafficking of neutrophils into the airspace. (A-D) Emp2 $2^{+/+}$and Emp2 $2^{-/-}$mice received the inhaled exposures shown and bronchoalveolar lavage (BAL) neutrophils (PMNs) were counted at various time points (A) or 24 hours after exposure (B-D) ( $n=5-7 / g e n o t y p e)$. (E) BAL fluid (BALF) cytokines and chemokines were quantified 2 hours after LPS inhalation ( $n=11-12$ /genotype). (F) Mice were administered CXCL1 to the lungs by oropharyngeal aspiration and BAL PMNs and BALF CXCL5 were quantified 4 hours later ( $n=5-6 /$ genotype). (G) Mice received intraperitoneal CXCL1 and peritoneal lavage PMNs were quantified 4 hours later ( $n=4$ /genotype). Data are the mean \pm SEM and are representative of at least 3 independent experiments. ${ }^{*} P<$ $0.05 ;{ }^{* *} P<0.01$ by unpaired 2-tailed Student's $t$ test.

multiple airway epithelial cell lines do express EMP2 (Supplemental Figure 3C), it is possible that EMP2 protein is expressed in the small airway epithelium in vivo but below the detection limit of our immunofluorescence antibody. Alternatively, the distribution of interstitial PMNs in the Emp2 $2^{-/}$lung could conceivably arise from abnormal intratissue PMN trafficking that initiates from a primary defect at the level of the terminal airspaces (e.g., necessitation up the bronchovascular sheath). Suggesting that epithelial EMP2 deficiency is sufficient to attenuate PMN TEM, we found a marked defect in migration of primary human PMNs in the physiological basolateral-to-apical direction through a monolayer of EMP2-silenced Calu-3 cells in response to the chemotactic peptide $N$-formyl-met-leu-phe (fMLP) (Figure 2E).

EMP2-deficient mice have improved survival during bacterial pneumonia. PMNs are critical for bacterial killing during pneumonia, but also cause deleterious bystander injury to the pulmonary epithelium (4). Indeed, overexuberant alveolar neutrophilia during pneumonia can compromise survival despite improving bacterial clearance $(17,18)$. To what degree PMNs eradicate bacteria in the alveolar lumen versus interstitium during pneumonia is unclear, but successful passage of bacteria through damaged pulmonary epithelium is associated with extrapulmonary dissemination and mortality (19). We found that there was no change in bacterial burden in whole (nonlavaged) lung homogenates of $E m p 2^{-/-}$mice during infection with K. pneumoniae (Figure $3 \mathrm{~A}$ ), and no consistent change in bacterial counts in BALF (not shown), collectively suggesting grossly intact bacterial clearance in the infected $E m p 2^{-/-}$lung. Of interest, Emp $2^{-/-}$mice, however, exhibited increased survival during lung infection with K. pneumoniae compared with WT counterparts (Figure 3B). This was associated with attenuated injury to the alveolocapillary barrier, as indicated by dramatic reductions in BALF protein, albumin, and IgM (Figure 3C).

Aiming to determine whether preservation of the epithelial barrier due to reduced PMN TEM was attenuating extrapulmonary bacterial dissemination in $E m p 2^{-/-}$mice, we surveyed the peripheral circulation. At 48 hours after infection, the time point of initial separation of survival curves between $E m p 2^{+/+}$and $E m p 2^{-/-}$mice (Figure 3B), Emp2 ${ }^{-/-}$mice had lower serum IL-6 and TNF- $\alpha$ (Figure 3D), denoting decreased systemic inflammation. However, there was no significant change in bloodstream or splenic bacterial burden (Supplemental Figure 7), arguing against reduced sepsis as an explanation for the decreased cytokines and mortality. Of note, Emp2 $2^{-/-}$mice displayed marked reductions in neutrophilic pulmonary consolidation and associated tissue damage on histopathologic analysis (Figure 3E and Supplemental Figure 8). This finding suggests that during pneumonia the lungs are the source of the differential serum cytokine levels in Emp2 $2^{+/+}$and $E m p 2^{-/-}$mice, and that $E m p 2^{-/-}$mice have relative protection from a respiratory death (i.e., respiratory failure from acute lung injury).

In order to test whether Emp2-/- mice might nonetheless be susceptible to pathogen overgrowth and dissemination after higher- 
A

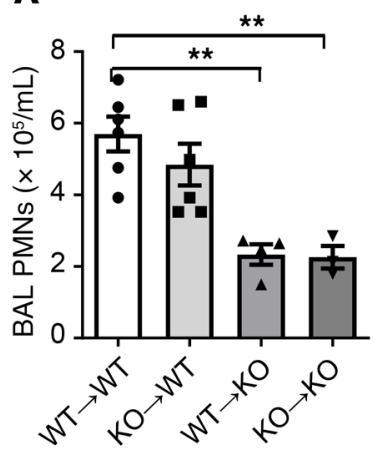

B

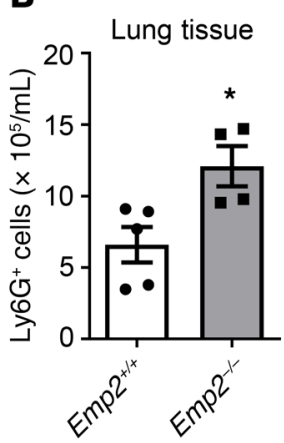

BAL

C

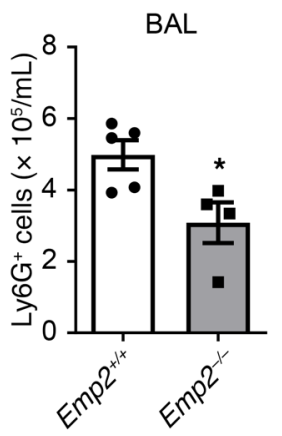

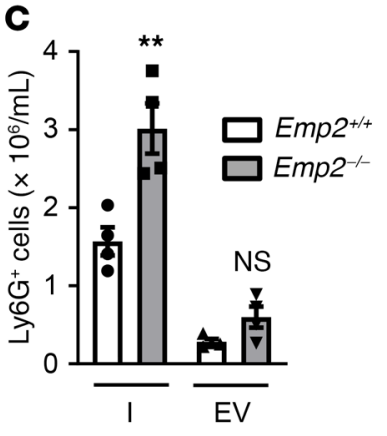

D

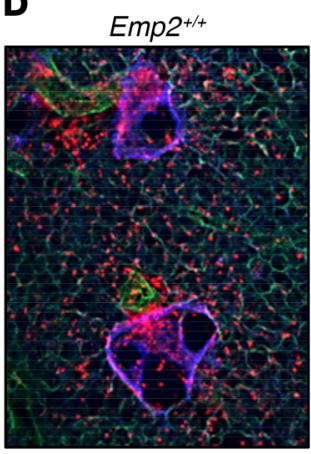

Epithelial cells

Endothelial cells

Neutrophils
E
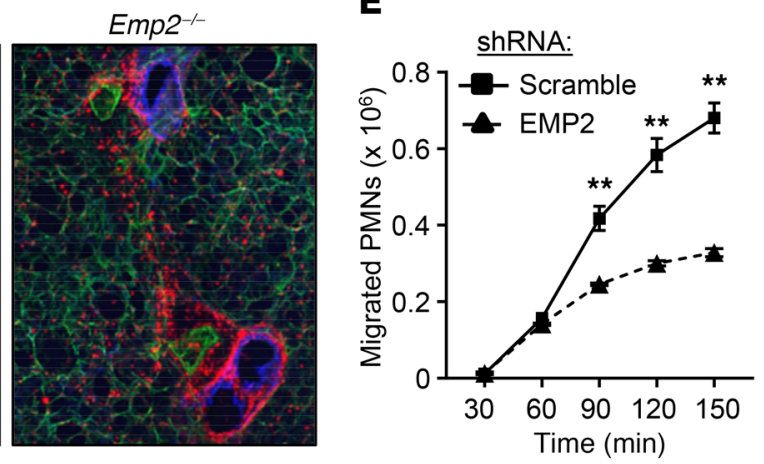

Figure 2. EMP2 regulates transepithelial migration of neutrophils into the alveolar lumen. (A) Bone marrow chimeric mice were made by transfer of bone marrow cells from $\mathrm{Emp2}^{+/+}$(WT) or Emp2 ${ }^{-l_{-}}$(KO) donors to $\mathrm{Emp2}^{+/+}$or $\mathrm{Emp2}^{-l_{-}}$irradiated recipients (donor[arrow]recipient). Chimeras were exposed to inhaled LPS, and BAL PMNs quantified 24 hours later ( $n=3-6 /$ chimera). (B) Eight hours after LPS inhalation, Ly6G+ PMNs were quantified by flow cytometry in lavaged and perfused lungs (left) and in the BAL (right) of $E m p 2^{+/+}$and $E m p 2^{-1-}$ mice ( $n=4-5 /$ genotype). (C) Pulmonary interstitial (I) and endovascular (EV) Ly6G $\mathrm{G}^{+}$PMNs were quantified under similar conditions to those in panel I ( $n=4 /$ genotype). (D) Live lung slices from Emp2 $2^{+/}$and $E m p 2^{-/-}$ mice were stained for E-cadherin (epithelium), CD31 (endothelium), and Ly-6G (PMNs) 6 hours after LPS. Emp2-/- lungs display an excess accumulation of peribronchovascular (interstitial) PMNs. Results are representative of $n=3-4 /$ genotype. (E) Human PMNs that transmigrated across a monolayer of scramble or EMP2 shRNA-transduced Calu-3 cells in response to fMLP during a time course were quantified ( $n=3 /$ condition/time point). Data are the mean \pm SEM and are representative of at least 3 independent experiments. ${ }^{*} P<0.05$; ${ }^{* *} P<0.01$ analyzed using ANOVA followed by Dunnett's test in $\mathbf{A}$, or unpaired 2-tailed Student's $t$ test in $\mathbf{B}, \mathbf{C}$, and $\mathbf{E}$.

burden infections, we next inoculated the lungs of Emp2 $2^{+/+}$and Emp2 $2^{--}$mice with $4000 \mathrm{CFU}$ K. pneumoniae. Of interest, at 48 hours after infection, we observed a significant reduction in bacterial burden in the lungs, bloodstream, and spleen of Emp2 $2^{-\digamma}$ mice (Supplemental Figure 9), suggesting enhanced pathogen clearance in the lungs and reduced extrapulmonary dissemination. Collectively, these findings suggest that antimicrobial host defense is impacted by EMP2 in a dose-dependent fashion, and that EMP2 deficiency does not compromise, but may rather enhance, pathogen clearance at higher infectious inocula.

EMP2 deficiency dysregulates epithelial display of adhesion molecules. We next sought to define the mechanism whereby EMP2 regulates transit of PMNs into the alveolus. Studies deriving from intestinal epithelium have suggested that PMN $\beta 2$ integrins initially engage fucosylated glycoprotein(s) on the basolateral epithelial surface, after which non- $\beta 2$ integrin PMN proteins interact with CD47 and other epithelial proteins during paracellular transit (6). Very few epithelial regulators of TEM have been specifically validated in the lung (4). av $\beta 3$ integrin, reportedly upregulated by EMP2 in cell lines (10), has been implicated in LPS-induced alve- olar neutrophilia (20). The $\beta 2$ integrin ligand ICAM-1, also upregulated by EMP2 in cell lines, has also been implicated in TEM in both the intestinal and alveolar lumen $(21,22)$.

As S. pneumoniae- and CXCL1-induced alveolar neutrophilia are reportedly fully, and LPS-induced neutrophilia, partially, CD18-independent $(3,23)$, we initially reasoned that dysregulation of CD18-independent ligands in Emp2 $2^{--}$epithelium was the most parsimonious explanation for our findings. However, given that LPSinduced alveolar neutrophilia is estimated to be only approximately 20\% CD18-independent (3), the marked PMN reduction observed in LPS- and Gram-negative bacteria-exposed Emp $2^{--}$lungs (Figure $1, \mathrm{~A}-\mathrm{C}$ ) suggested that dysregulation of ligands for both $\mathrm{CD} 18$ and other PMN proteins was likely present in Emp2 $2^{-/}$epithelium.

Using flow cytometry, we found that surface display of CD47 was increased on the AT1 cells of Emp2-- mice, whereas ICAM-1 and $\beta 3$ integrin were reduced (Figure 4A and Supplemental Figure 10), collectively indicating wide-ranging dysregulation of adhesion molecules on the AT1 plasma membrane. As perhaps expected given that AT2 cells do not express EMP2 protein (Supplemental Figure 2), surface levels of the 3 proteins were equiv- 
A

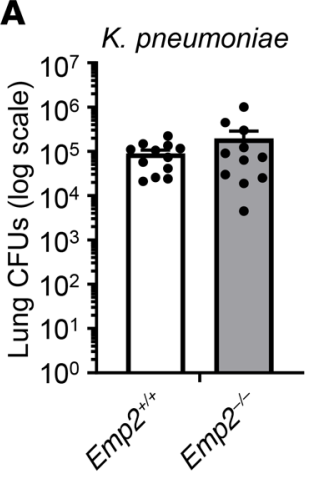

C
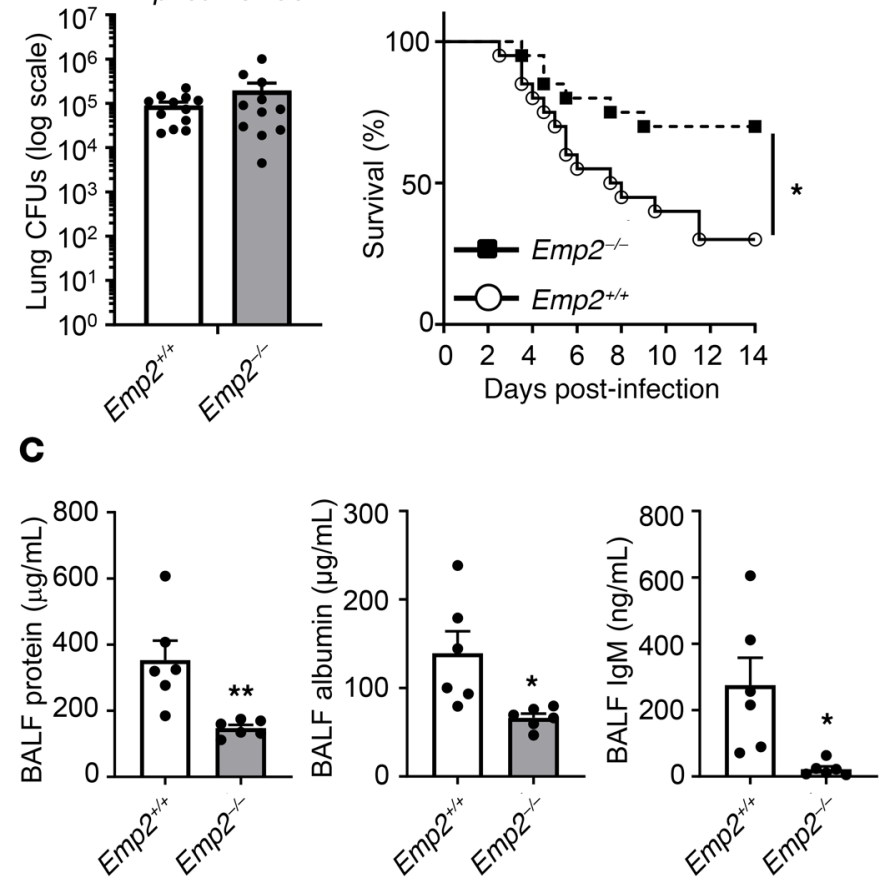

D

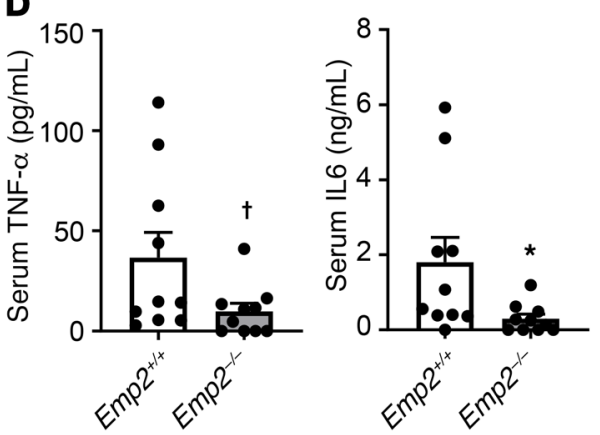

E

$E^{\prime \prime m p 2+/+}$
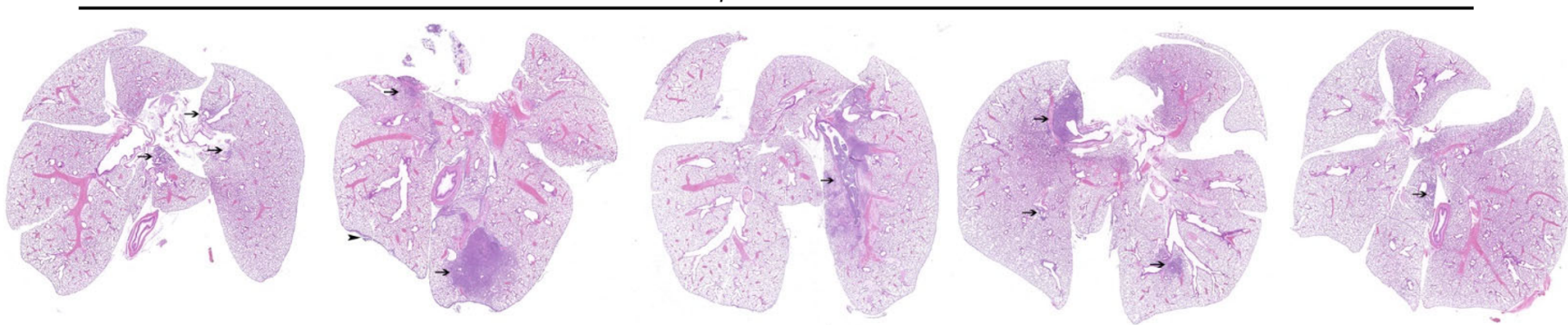

Emp2--
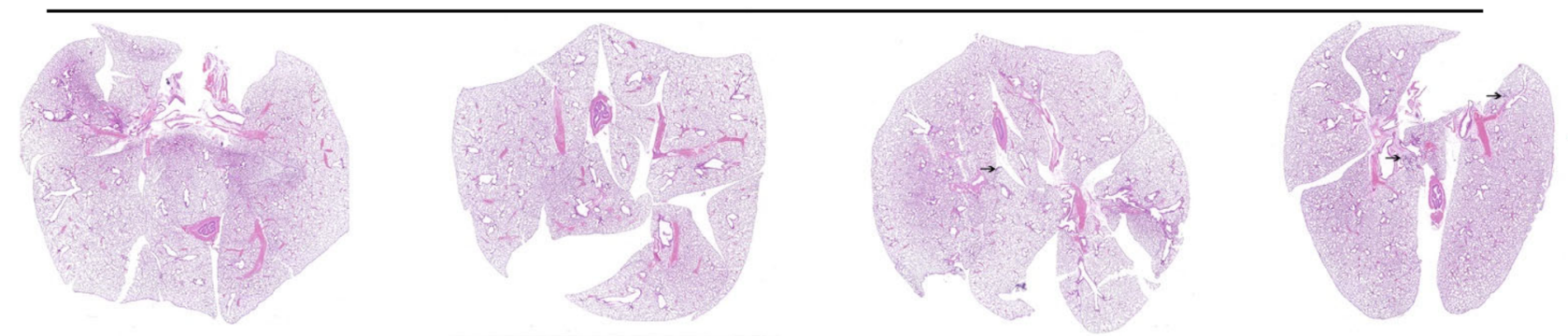

Figure 3. EMP2-null mice have reduced mortality and lung injury during bacterial pneumonia. (A) $E m p 2^{+/+}$and $E m p 2^{-/-}$mice were infected with $K$. pneumoniae by oropharyngeal aspiration and then had bacterial CFUs quantified in lung homogenates 24 hours after infection ( $n=11-12 /$ genotype). (B) Survival was monitored in mice infected with $K$. pneumoniae as in $\mathbf{A}(n=20$ /genotype). (C) BALF protein, albumin, and IgM were measured in mice 48 hours after K. pneumoniae inhalation ( $n=5-6 /$ genotype). (D) Serum TNF- $\alpha$ and IL-6 were quantified by ELISA 48 hours after lung infection with $K$. pneumoniae. (E) Representative images of lungs from mice ( $n=4-5$ /genotype; $\times 1$ magnification) 48 hours after infection with $K$. pneumoniae by oropharyngeal aspiration. Inflammatory areas are indicated by arrows; arrowhead points to inflammation on the pleural surface. Inflammation (predominantly neutrophilic infiltration) was more severe in $\mathrm{Emp2}^{+/+}$mice. Data in $\mathbf{A}, \mathbf{C}$, and $\mathbf{D}$ are mean $\pm \mathrm{SEM}$ and are representative of at least 3 independent experiments. ${ }^{\dagger} P=0.06$; ${ }^{*} P<$ $0.05 ;{ }^{*} P<0.01$ by unpaired 2-tailed Student's $t$ test or log-rank test (survival).

alent between $E m p 2^{+/+}$and Emp2 $2^{-/-}$AT2 cells. EMP2 silencing in both Calu-3 cells and H292 cells largely recapitulated the adhesion molecule changes in primary AT1 cells (Figure 4, B and C), suggesting that Calu-3 cells are an appropriate cell line with which to model mechanisms of impaired TEM across Emp $2^{-/-}$AT1 cells.
EMP2 is required for AT1 cell raft integrity. EMP2 localizes to lipid rafts in cell lines and has been proposed to support lipid raft assembly and to specify proteins for rafts by acting as a scaffolding protein $(9,11)$. The multiple adhesion molecule changes in Emp2 $2^{-/}$AT1 cells, including proteins that localize 
A
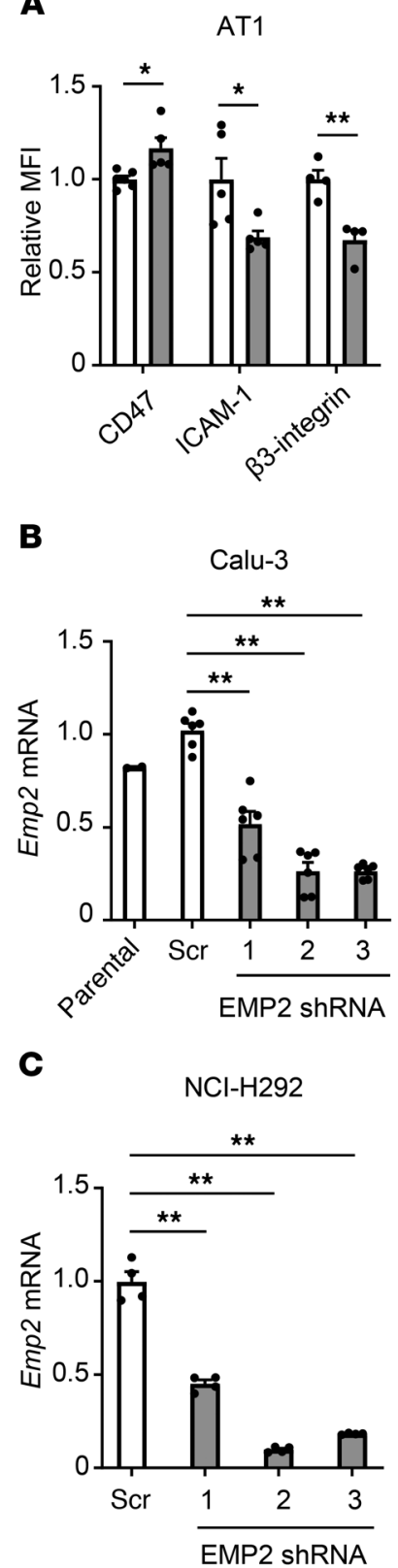

AT2
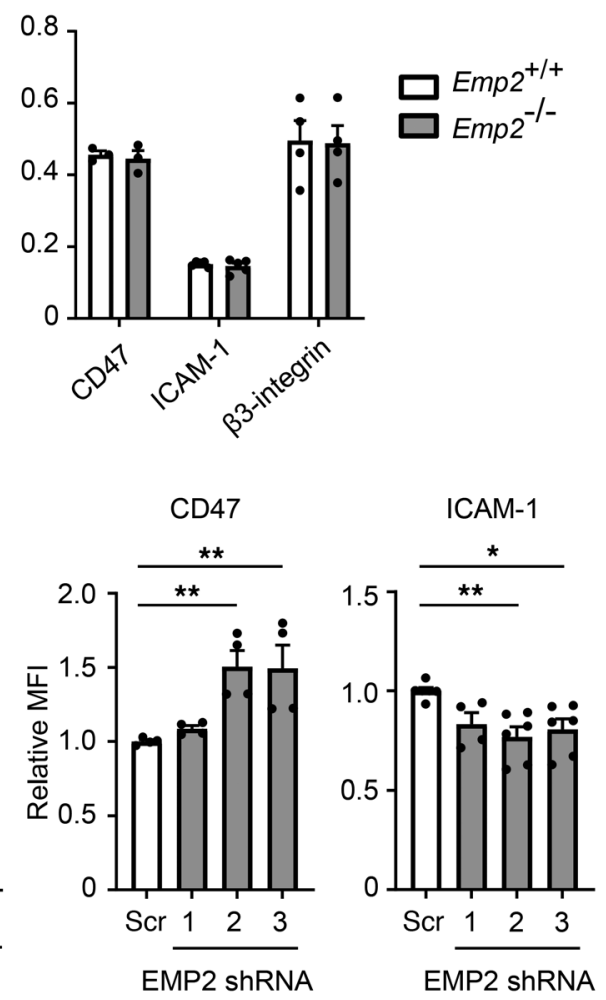

$\beta 3$-integrin
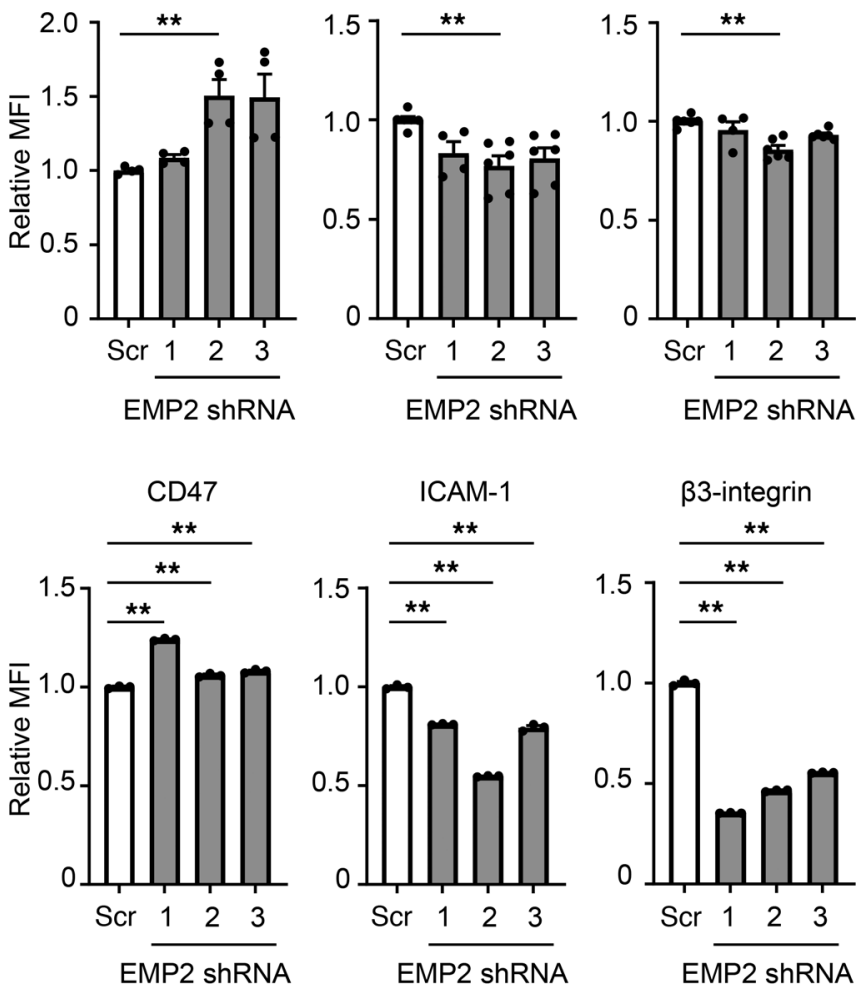

Figure 4. EMP2 deletion dysregulates epithelial surface display of adhesion molecules. (A) CD47, ICAM-1, and $\beta 3$ integrin were quantified by flow cytometry on AT1 (CD45-CD31-CD34 EpCAM ${ }^{\text {int }}{ }^{T} 1 \alpha^{+} \mathrm{MHCII}^{-}$) and AT2 (CD45-CD31-CD34-EpCAM ${ }^{\text {int }}$ T1 $\alpha-\mathrm{MHClI}^{+}$) cells from naive $E m p 2^{+/+}$and $E m p 2^{-1-}$ mice. (B and C) Calu-3 (B) and H292 (C) cells underwent lentiviral transduction with either scramble (scr) or EMP2-targeting shRNAs. Emp2 mRNA was quantified by $\mathrm{qPCR}$, and surface CD47, ICAM-1, and $\beta 3$ integrin were quantified by flow cytometry. Data are the mean \pm SEM and are representative of at least 3 independent experiments. ${ }^{*} P<0.05$; ${ }^{*} P<0.01$ analyzed using unpaired 2-tailed Student's $t$ test in A, or ANOVA followed by Dunnett's test in B and C.

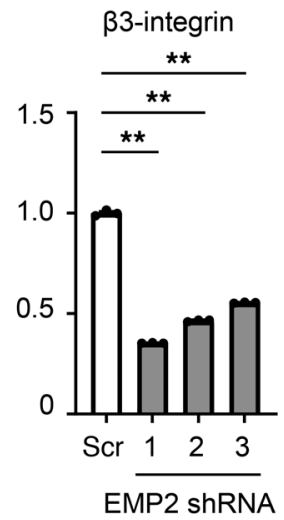

to rafts $(4,8)$, suggested to us raft dysregulation as a potential unifying mechanism. Consistent with this, we found that primary murine AT1-like cells transdifferentiated in vitro from mouse lung digests displayed a significant reduction in surface binding of cholera toxin B (CtB) (Figure 5A), a ligand for GM1 and other raft-localized gangliosides that has been used widely as a metric for raft mass (13). Changes in CtB signal of similar or smaller magnitude in other cell systems have been shown to track with biologically significant changes in raft function $(24,25)$. Raft mass was similarly reduced in EMP2silenced Calu-3 cells (Figure 5, B and C) and H292 cells (Figure 5D). Conversely, in Let1 cells, an AT1-like cell line (26) in which we could not detect expression of native EMP2 protein by immunoblot (not shown), overexpression of EMP2 augmented $\mathrm{CtB}$ binding, suggesting an increase in raft mass (Figure $5 \mathrm{E}$ ).
Localization of EMP2 to rafts has been reported to be cholesterol-dependent $(9,11)$, suggesting that direct or indirect interactions of EMP2 with raft cholesterol may be necessary for its raft-scaffolding functions. Cholesterol recognition/interaction amino-acid consensus (CRAC) motifs, with the sequence $\mathrm{L} / \mathrm{V}-\mathrm{X}_{1-5^{-}}$ $\mathrm{Y}-\mathrm{X}_{1-5}-\mathrm{K} / \mathrm{R}$ (where $\mathrm{X}_{1-5}$ is 1 to 5 of any amino acid and the central tyrosine is proposed to be critical) and inverted CRAC ('CARC') motifs are 2 amino acid sequences by which membrane proteins are proposed to bind to cholesterol (27). Both motifs are proposed to stabilize cholesterol in membranes to support assembly of rafts (28). The tetraspan family member PMP22 has a CRAC motif in its fourth transmembrane domain (29). Given this, we manually inspected EMP2, and noted it to have one potential CRAC sequence in, plus 2 potential CARC sequences near, its predicted fourth transmembrane domain (Supplemental Figure 
A

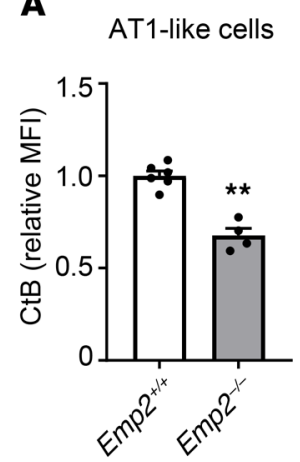

C
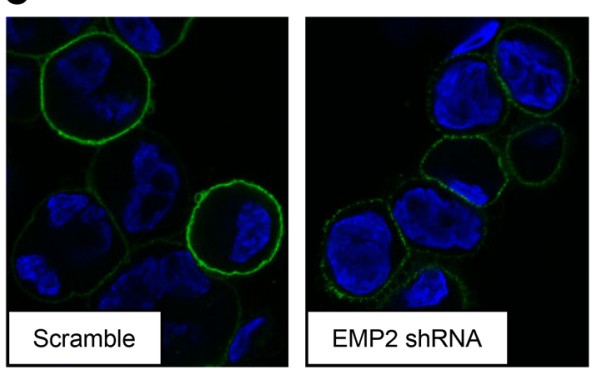

D

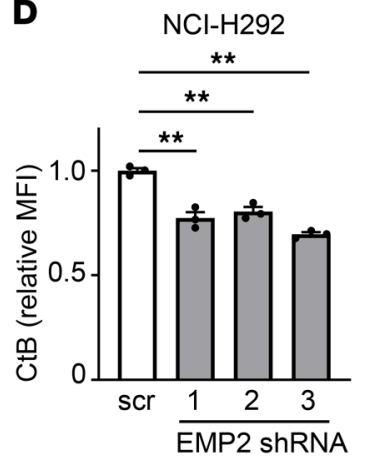

Figure 5. EMP2 deletion depletes lipid rafts in epithelial cells. (A) Primary alveolar epithelial cells were purified from $\mathrm{Emp2}^{+/+}$and $\mathrm{Emp2}^{-I^{-}}$lungs and cultured for 8 days to produce AT1-like cells (see Supplemental Figure 3C). Lipid rafts were then quantified by flow cytometry of bound cholera toxin B (CtB)-Alexa Fluor 488. (B and C) Calu-3 cells were transduced with scrambled (scr) or EMP2-targeting lentiviral shRNAs, after which CtB binding was quantified by flow cytometry (B) and imaged by fluorescence microscopy (CtB-Alexa Fluor 488 = green; DAPI = blue) (C). Original magnification $\times 630$. (D) H292 cells were transduced and analyzed for CtB binding as in B. (E) Let1 cells were transfected with EMP2 or empty vector. CtB binding by flow cytometry is shown at left, and relative Emp2 mRNA by qPCR at right. (F) Let1 cells were transfected with WT EMP2 or with EMP2 mutated in its CRAC or CARC sequences (see Supplemental Figure 11). Cells were then analyzed as in E. Data are mean \pm SEM and are representative of at least 3 independent experiments. ${ }^{*} P<0.05$; ${ }^{* *} P<0.01$ analyzed using unpaired 2-tailed Student's $t$ test in $\mathbf{A}$ and $\mathbf{E}$, or 1-way ANOVA followed by Dunnett's test in $\mathbf{B}, \mathbf{D}$, and $\mathbf{F}$.
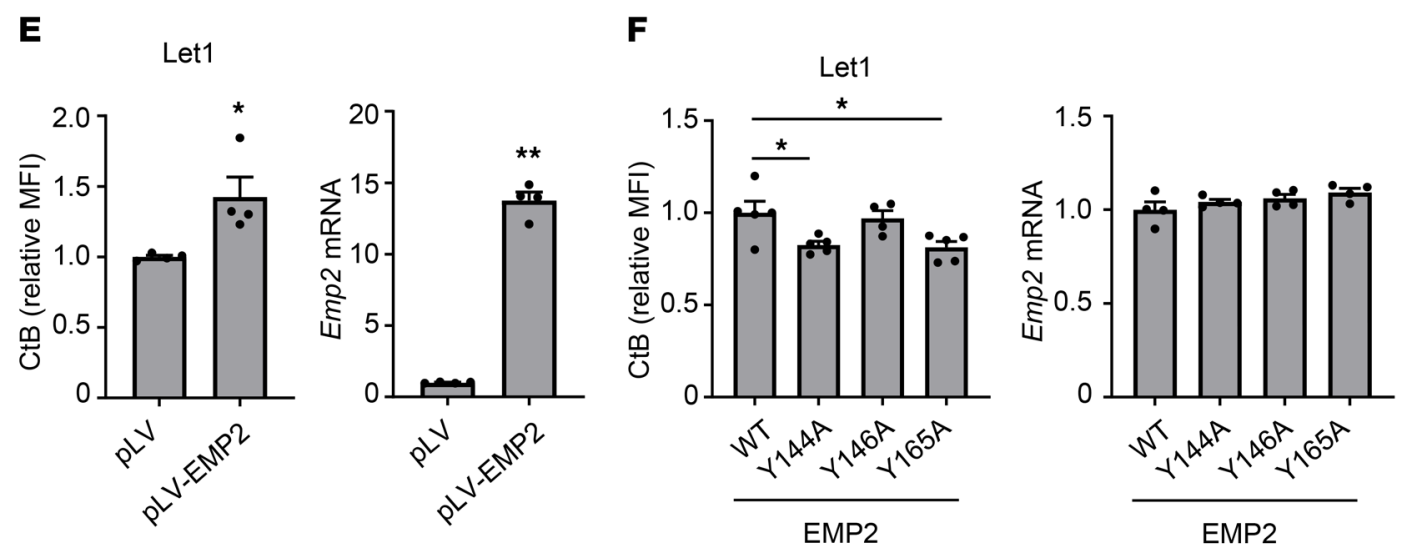

11). Aiming to test whether one or more of these sequences are required for EMP2 support of raft assembly, we mutated the central tyrosine residue of the 2 CARCs nearest the transmembrane domain (Y144, Y146), as well as that of the CRAC motif (Y165), and expressed these mutants along with WT EMP2 (EMP2-null) in Let1 cells. Notably, the Y144A and Y165A EMP2 mutants failed to support CtB binding in Let1 cells to the degree of WT EMP2 (Figure 5F). This suggests that interactions of EMP2 with cholesterol in its fourth transmembrane domain are required for its raftsupporting function in AT1 cells, potentially through promoting EMP2 localization to rafts. By contrast, although overexpression of WT EMP2 increased ICAM-1 surface display in Let1 cells, equivalent upregulation was observed with the CARC and CRAC mutants (Supplemental Figure 12), suggesting that, at least in this cell line and overexpression system, cholesterol interactions may not be required for EMP2 support of adhesion molecule display.

EMP2 regulates epithelial adhesion molecules and TEM through caveolin-2. From its location in rafts, EMP2 is thought to act in trans on caveolae, distinct membrane microdomains whose flask-like shape is driven by hetero-oligomerization of the membrane proteins caveolin-1 and -2 (30). Through somewhat obscure mechanisms, EMP2 reduces expression of both caveolins (9). Caveolins, like EMP2, regulate other membrane proteins through scaffolding and signaling interactions (30). In renal podocytes, EMP2 silencing induces cell injury through upregulation of caveolin-1 (31). Given this, we hypothesized that in AT1 cells, EMP2 deficiency might alter adhesion molecules and PMN TEM via dysregulation of the caveolins.

Consistent with prior reports in Emp2-silenced cell lines (9), we found that caveolins-1 and -2 were upregulated in Emp $2^{-/-}$lung homogenates (Figure 6A). Although the upregulation was modest, this may not be unexpected given our finding that EMP2 is expressed in only a subset of pulmonary cells. A more robust upregulation was indeed confirmed upon EMP2 silencing in Calu-3 cells (Figure 6B). Conversely, ectopic expression of EMP2 in CMT 64 cells downregulated both caveolins (Figure 6C). 
A

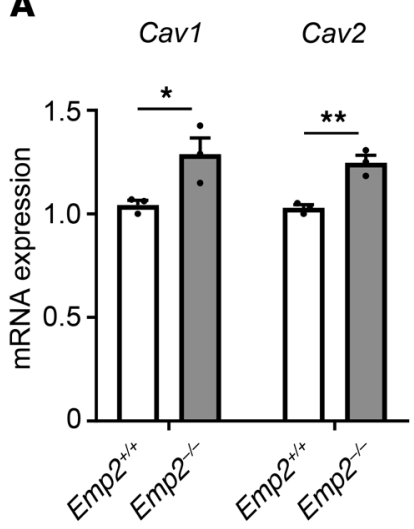

D

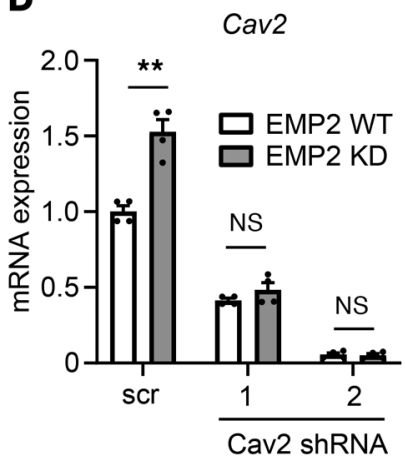

B

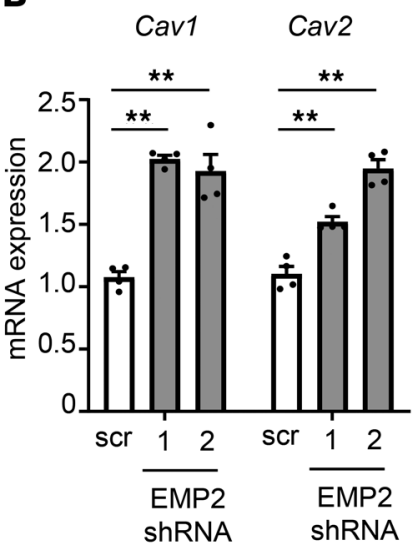

C
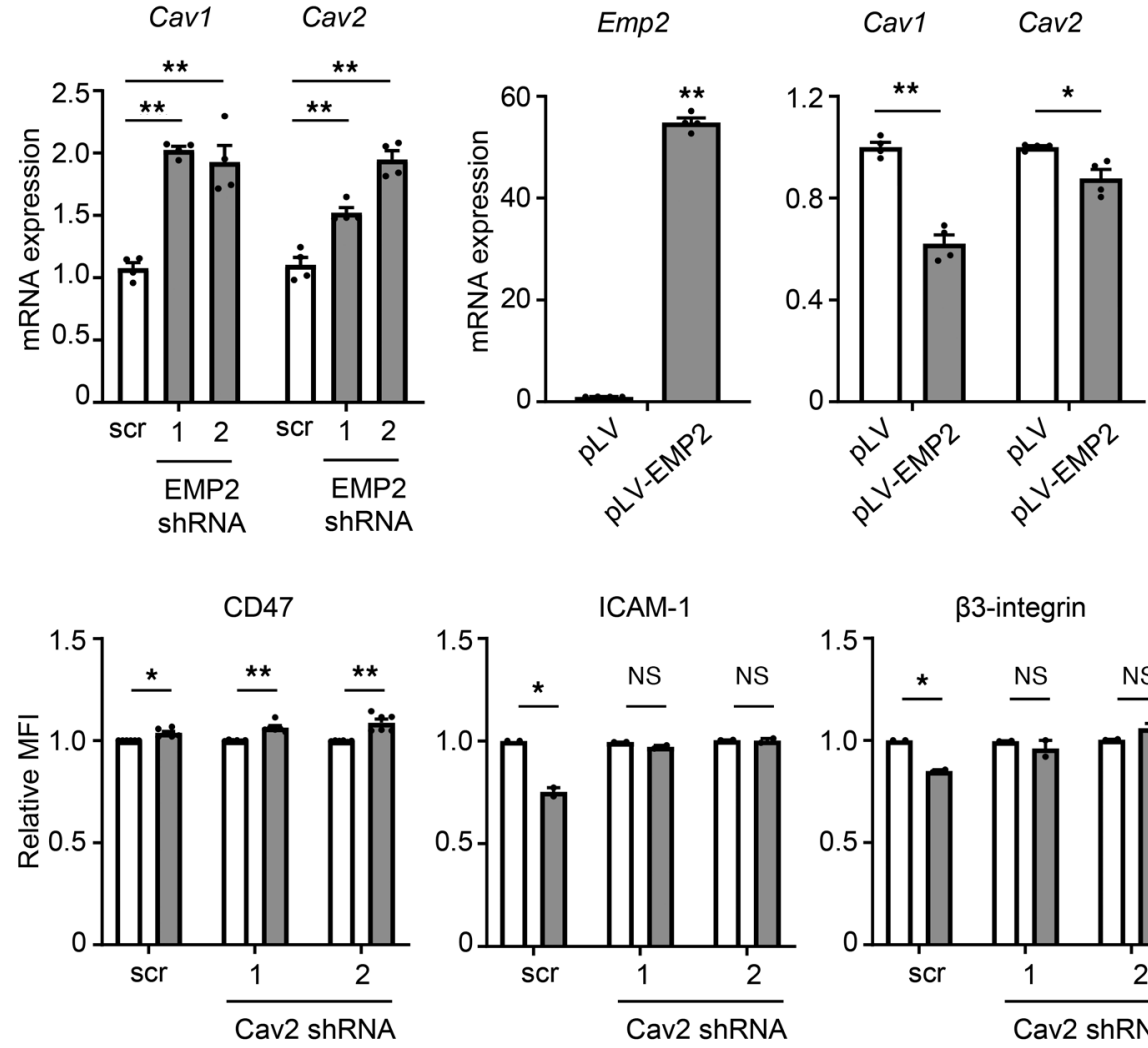

B3-integrin

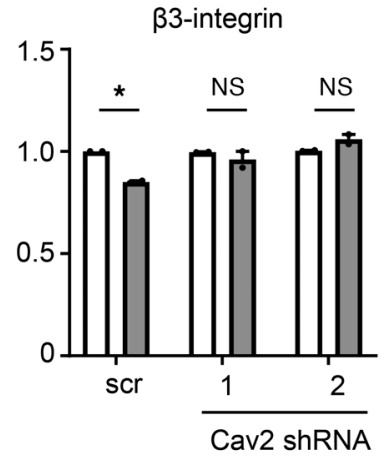

$\mathbf{E}$
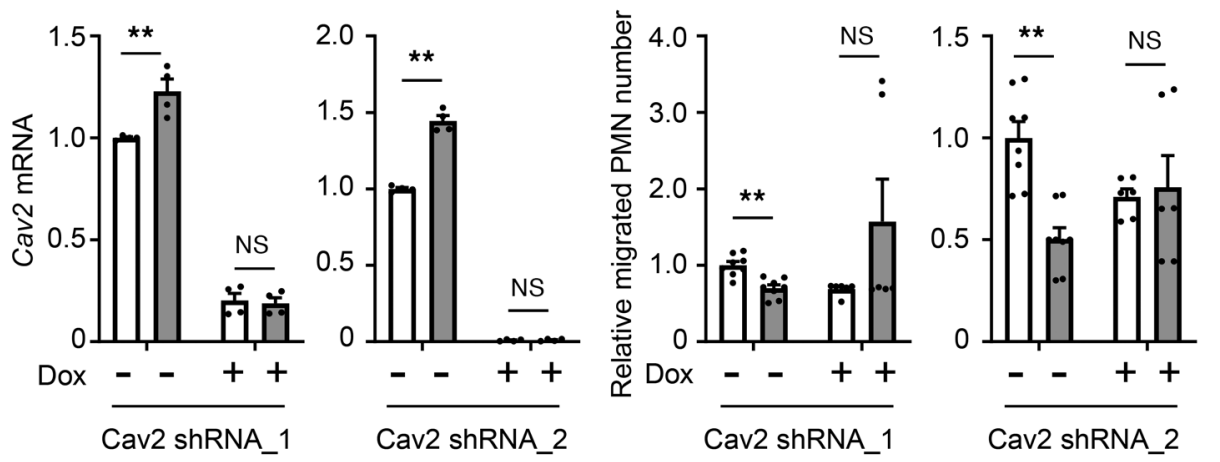

$\square$ EMP2 WT
$\square$ EMP2 KD

Figure 6. EMP2 regulates adhesion molecules and TEM through caveolin-2. (A) Caveolin 1 (Cav1) and Cav2 mRNA was quantified by RT-qPCR in lungs of naive $\mathrm{Emp2}^{+/+}$and Emp2-/- mice ( $n=3 /$ genotype). (B) Cav1 and Cav2 mRNA was quantified by RT-qPCR in Calu-3 cells after stable EMP2 silencing with 2 lentiviral shRNA constructs, or treatment with scrambled (scr) lentiviral shRNA control (4 biological replicates). (C) EMP2 was ectopically expressed in CMT 64 cells; empty vector served as a control. Emp2, Cav1, and Cav2 were then quantified by RT-qPCR (4 biological replicates). (D) Stable EMP2 knockdown (KD) or scrambled control (WT) Calu-3 cells underwent lentiviral shRNA silencing of Cav-2, or parallel treatment with scr vector. Cav2 mRNA was quantified by RT-qPCR (left). Cell surface CD47, ICAM-1, and $\beta 3$-integrin were quantified by flow cytometry and are depicted as relative MFI (right). (E) Stable EMP2 KD or WT Calu-3 cells were transduced with doxycycline-inducible (dox-inducible) Cav-2 shRNA constructs and then left untreated or treated with doxycycline. Cav-2 silencing was confirmed by RT-qPCR (left). Human neutrophil (PMN) transmigration to fMLP across Cav-2-silenced and unsilenced monolayers was quantified (right; $n=6-8$ wells per condition, normalized across 2 independent experiments). Data are mean \pm SEM. A-D are representative of at least 3 independent experiments. ${ }^{*} P<0.05 ;{ }^{* *} P<0.01$ analyzed using unpaired 2-tailed Student's $t$ test in $\mathbf{A}$ and $\mathbf{C}-\mathbf{E}$, or 1-way ANOVA followed by Dunnett's test in $\mathbf{B}$.

Using Calu-3 cells as a model system, we sought to test whether the effects of EMP2 deficiency we had identified in epithelial cells were dependent upon caveolins. Although the 2 caveolins have distinct functions, the lung abnormalities of $\mathrm{Cav1}^{--}$mice (i.e., hypercellularity) have been attributed to caveolin-2 deficiency (32). Given this, and our finding that Calu-3 cells express robust caveolin- 2 but not caveolin-1 protein on immunoblot (not depicted), we focused our interventions on caveolin-2. Caveolin-2 silencing by shRNA abolished the relative upregulation of this protein observed in EMP2deficient Calu-3 cells (Figure 6D). Of interest, caveolin-2 silencing rescued the reduced surface display of both ICAM- 1 and $\beta 3-$ integrin observed in EMP2-deficient Calu-3 cells but did not reverse their modest upregulation of CD47 (Figure 6D). This find- 
ing suggests that some but not all of the membrane-regulatory effects of EMP2 are mediated via caveolins. Whether this reflects distinct membrane microdomain (i.e., caveolar) partitioning between CD47 and ICAM-1/ $\beta 3$-integrin or an alternate mechanism remains unclear.

In order to test more directly whether EMP2 regulates PMN TEM in a caveolin-dependent fashion, we engineered doxycycline-inducible caveolin-2-silenced Calu-3 cells on both the EMP2-sufficient and -deficient background and cultured them to confluence for PMN transmigration studies. Both caveolin-2 silencing constructs depleted caveolin-2, neutralizing its relative upregulation in EMP2-deficient cells (Figure 6E). Of interest, the caveolin-2 silencing constructs augmented PMN transit through EMP2-deficient Calu-3 monolayers. The reduction in PMN transmigration observed with EMP2 knockdown was no longer seen in caveolin-2-silenced Calu-3 cells (Figure 6E). This finding indicates that the reduction in PMN TEM observed in EMP2-deficient cells is caveolin-dependent.

\section{Discussion}

Trafficking of PMNs to the lung has been studied for decades, but the extent to which the pulmonary epithelium serves as a final gatekeeper to the airspace for PMNs that have exited the vasculature, as well as the degree to which epithelial control of PMN trafficking is a regulated and coordinated event, has remained largely undefined. Here, we identify EMP2, a gene highly expressed in the lung but previously of unknown function in lung biology, as a master regulator of the terminal entry of PMNs into the airspace lumen. Our studies suggest that EMP2 supervises display of adhesion molecules on AT1 cells through organization of membrane microdomains, thereby ensuring cognate interactions for PMNs in transit.

Planar rafts and flask-shaped caveolae are distinct cholesterolenriched membrane microdomains that both serve as platforms for protein-protein and lipid-protein interactions (13). Caveolae, whose invaginated morphology derives from caveolin-1/-2 heterodimers, are found in a subset of cell types (e.g., AT1 cells, endothelial cells, fibroblasts). In these cells, it is thought that caveolae coexist with spatially segregated rafts, but the relative function, protein repertoire, and crosstalk between these 2 microdomains remain poorly understood. Indeed, given that membrane fractionation methods fail to distinguish between rafts and caveolae, and sophisticated techniques such as immunogold electron microscopy are instead required, many reports have almost certainly conflated the 2 microdomains (13). In the case of EMP2, it has been shown that it does not colocalize with caveolae or caveolins, but rather is specific for rafts, from where it supports the levels of other raft proteins, likely through scaffolding or other interactions (9).

Of interest, caveolin-1 is not only necessary, but also sufficient for biogenesis of caveolae, as heterologously expressed caveolin-1 binds and clusters specific membrane lipids (33), inducing formation of invaginated caveolae (34). Upregulation of caveolin-1 dysregulates endocytosis of caveolae (35), suggesting that the function of existing caveolae is also sensitive to changes in caveolin-1 expression. In turn, several proteins have been identified that modify caveolae through direct or indirect effects on caveolins (35-37). Among these, EMP2 reportedly suppresses expression of caveolin-1 and -2 at the mRNA and protein level (9), thereby impacting caveolin-dependent signaling (38). The protein-level effect may be through lysosomal delivery of caveolin-1 (38). Taken together, EMP2 appears to coordinate different membrane microdomains, reciprocally supporting localization of proteins to rafts and suppressing caveolar signaling.

Here, we show for what we believe is the first time that EMP2 support of raft mass (i.e., GM1 signal) is contingent upon 2 putative cholesterol-binding sequences that are predicted to lie in or near its fourth transmembrane domain. This finding suggests that EMP2 may serve as a molecular scaffold that physically intermediates association of cholesterol with proteins in rafts. Our studies were not designed to discriminate the raft versus caveolar localization of adhesion molecules in AT1 cells, nor does cell-surface display as quantified by flow cytometry clarify whether proteins have been displaced from their native microdomains. Our finding that CD47 dysregulation in EMP2-deficient cells was independent of caveolin-2 is consistent with prior reports that CD47 is raftresident (18). Conversely, the caveolin-2 dependence of ICAM-1 dysregulation that we observed in EMP2-deficient cells is consistent with its reported caveolar localization (30); the inverse relationship we found between it and caveolin is reminiscent of the caveolar protein HER2, whose cell-surface levels are reportedly reduced by caveolin- 1 overexpression and stabilized by caveolin- 1 silencing (39). Finally, our finding that $\beta 3$ integrin is downregulated in EMP2-null epithelial cells is consistent with prior reports that EMP2 and $\alpha \mathrm{v} \beta 3$ are physically associated in endometrial cell membranes (10); however, the caveolin-2 dependence of this downregulation, which we observed, is perhaps unexpected.

We speculate that EMP2 coordinates adhesion molecule display in different membrane microdomains of AT1 cells through both direct and indirect mechanisms. Although we originally aimed to test whether mutagenesis of CRAC/CARC sequences in murine EMP2 impairs PMN TEM, we were unable to do so, due to the failure of 2 EMP2-deficient murine alveolar epithelial cell line (Let1, CMT64) monolayers to culture tightly enough to support an effective chemokine gradient (not depicted). In the end, our Calu3 system indicates that the deficit in TEM conferred by EMP2 deficiency is caveolin-2 dependent and that it tracks with downregulation of ICAM- 1 and $\beta 3$ integrin display. The magnitude of change in individual adhesion molecules in EMP2-deficient cells is modest. We speculate, however, as is supported by the TEM studies, that, together, the coordinate dysregulation of multiple surface proteins is sufficient to compromise interactions of epithelial cells with migrating PMNs. Of potential interest, occludin, which we found to be reduced in naive $E m p 2^{-/-}$lung homogenates, has also been reported to support TEM (40). As PMN TEM is not abolished in Emp $2^{-/-}$mice, we conclude that EMP2 is not indispensable for PMN transit, but instead serves as a modifier, tuning cell traffic through its effects on the AT1 membrane. Given the importance of PMNs to environmental responses, it is perhaps unsurprising that redundancy is built into PMN trafficking. We speculate that additional raft scaffolding proteins (e.g., flotillins [refs. 13, 41]) may contribute to adhesion molecule display in AT1 cells. In support of this, flotillin-2 is reported to regulate integrin trafficking in epithelial cells (42). Future studies using targeted proteomics may be 
warranted to better define the extent of changes in the membrane protein complement of EMP2-null epithelial cells, and the subset of these changes that are either mediated via the caveolin proteins and/or sensitive to CRAC/CARC mutagenesis.

The finding that $E m p 2^{-/-}$mice have reduced intra- and extrapulmonary bacterial burden after lung infection with a large inoculum (4000 CFU; Supplemental Figure 9) is particularly intriguing. As lung and bloodstream bacterial counts were equivalent between $E m p 2^{+/+}$and Emp2 $2^{-/-}$mice at lower inocula, we conclude that there is a pathogen threshold - and associated lung injury threshold - above which EMP2 deficiency augments host defense. Reduced PMN-dependent injury to the respiratory epithelium in high inoculum-infected Emp $2^{-/-}$mice may improve bacterial compartmentalization in the lung, decreasing extrapulmonary dissemination. While the explanation for improved bacterial clearance in the high inoculum-infected Emp2 $2^{-/-}$lung is less evident, we speculate that it too may arise from improved compartmentalization in the airspace. This may allow for more efficient in situ killing in the alveolus, perhaps further buttressed by increased numbers of subepithelial (interstitial) PMNs (Figure 2, B and C) positioned to address bacteria that do penetrate the epithelium.

Caveolins are widely expressed in the murine lung, including in AT1 cells, endothelial cells, alveolar macrophages, and infiltrating PMNs (43). Caveolin-1-null mice, which are also caveolin-2 deficient (44), have abnormal pulmonary histopathology $(44,45)$ and reduced lung injury in response to LPS (46). To our knowledge, PMN TEM has not been studied in these mice; instead, their pulmonary phenotypes have been largely ascribed to changes in endothelial (47) and PMN (48) functions. Our findings in Emp2-/mice (which we show have caveolin upregulation in the lung), suggest that future studies of mice with pulmonary epithelial-targeted caveolin overexpression may be warranted.

Our findings do not rule out potential roles for EMP2 in nonepithelial cell types of the lung. Although EMP2 is expressed by NIH 3T3 fibroblasts (7), we did not evaluate EMP2 expression by pulmonary fibroblasts. PMNs are thought to extensively contact fibroblasts and ECM molecules during migration through the pulmonary interstitium (2). Given this, we cannot formally exclude the possibility that interstitial accumulation of PMNs in the Emp2-1lung arises in part from altered integrin ligand display on interstitial fibroblasts or altered ECM composition. Mouse lung endothelial cells express EMP2 mRNA (http://bis.zju.edu.cn/MCA/search. html). However, the increase of interstitial PMNs in LPS-exposed $E m p 2^{-/-}$lung, taken together with the decrease in alveolar PMNs, argues against dysregulated endothelium as a sufficient cause for the PMN trafficking defect. In the setting of $K$. pneumoniae, the overall reduction in PMNs we observed in Emp2 $2^{-/-}$lungs 48 hours after infection (Figure 3E) could conceivably arise from dysregulated endothelial adhesion molecules; thus, future studies involving cell-specific Emp2 deletion may be warranted to resolve whether endothelial EMP2 contributes to PMN trafficking in some settings (e.g., live pathogen exposures, later time points). Finally, although we found no detectable expression of EMP2 by AMs in the naive murine lung and our bone marrow chimera data point to EMP2 deficiency in nonhematopoietic cells as responsible for the PMN trafficking deficit, our data do not exclude the possibility that EMP2 may be upregulated in AMs during chronic inflammation and car- ry out roles in that cell type. Indeed, a recent report indicates that EMP2 is upregulated in macrophages by mycobacteria (49).

We speculate that EMP2 may regulate much wider-ranging functions of AT1 cells in lung biology, and may perhaps have been evolutionarily selected for these other functions, given that $E m p 2^{-/-}$mice fare better during bacterial pneumonia. Given that alveolar epithelial cells adhere to and migrate upon fibronectin via $\alpha v \beta 3$ (50), EMP2 may support epithelial repair in the injured lung. EMP2 may also modify AT1 adhesive preference for ECM through control of integrin trafficking, as has been reported in cell lines (7), and may thus alter outside-in signaling from the ECM in AT1 cells. In HEK293 cells, EMP2 binds to and augments function of P2X7 (51), a purinergic receptor that in AT1 cells interacts with claudin-18 to potentially regulate the alveolar epithelial barrier (52). Whether AT1 EMP2 regulates the alveolar epithelial barrier through interactions with P2X7 warrants investigation. Given that caveolins and caveolae play key roles in cell signaling, viral entry, and cell tolerance to mechanical stress (53), EMP2 may conceivably regulate these key events in AT1 cells through control of caveolin expression. Finally, given that other leukocyte types (i.e., eosinophils, monocytes, lymphocytes) also engage adhesion molecules during transit into the airspace, it will also be important to test whether EMP2 regulates trafficking of these cells during disease, perhaps supporting beneficial cell trafficking in response to some environmental insults.

In the mouse uterus, EMP2 localizes to the apical (luminal) surface of endometrial cells at the time of blastocyst implantation $(10,45)$ and is thus accessible to EMP2-blocking diabodies delivered locally via the intravaginal route (15). Anti-EMP2 antibodies are presently under preclinical investigation for cancer indications in humans (54). We found that i.t. pretreatment of WT mice with anti-EMP2 $\mathrm{scF}$ v reduced LPS-induced airspace neutrophilia (Supplemental Figure 5). Although the effect of EMP2 blockade on neutrophilia was modest under the conditions that we tested, further optimization may be possible. We propose that EMP2 targeting in the lung, potentially with the use of topical (inhaled) agents, may possibly offer a novel, selective therapeutic strategy against lung diseases driven by acute (acute lung injury), chronic (chronic obstructive pulmonary disease), or recurrent (bronchiectasis) excessive PMN delivery to the airspace. Unlike agents under development that block PMN trafficking indiscriminately (e.g., CXCR2 antagonists), such a strategy should avoid systemic immunosuppression. EMP2 mutations have recently been identified that underlie childhood-onset nephrotic syndrome (55), and may do so through caveolin dysregulation (31). Given this, we propose that genetic variation in EMP2 should now also be examined in relation to human lung disease.

Taken together, our findings suggest that AT1 cells, heretofore primarily considered in the context of gas exchange and ion transport, play an important role in regulating the final step of PMN delivery to the infected airspace. Interestingly, EMP2 supports this transfer of PMNs, but, at least in some contexts, at critical cost to epithelial barrier integrity and survival of the host.

\section{Methods}

Mice. Emp2 $2^{-/-}$mice have been described (14) and were on a C57BL/6 background. Age- and sex-matched WT littermate controls were used. In a subset of experiments, C57BL/6 controls were used (Jackson Laboratory). 
Mouse exposures and harvests. Mice were administered 150-2000 CFU K. pneumoniae (ATCC 43816), $2 \times 10^{5}$ CFU S. pneumoniae (serotype 3, ATCC 6303), or $1 \times 10^{6}$ CFU P. aeruginosa (PAO1, ATCC HER1018), or $0.5 \mu \mathrm{g}$ CXCL1 (R\&D Systems) by oropharyngeal aspiration while under flow-regulated isoflurane anesthesia. In some experiments, mice received $250 \mu \mathrm{g}$ of either anti-EMP2 scFv KS83 (Genscript) (15) or mouse IgG Fab fragment (Jackson ImmunoResearch) to the lungs by oropharyngeal aspiration. For microbiological analysis, whole lung was homogenized in $1 \times$ PBS, and serial dilutions plated on blood agar for bacterial quantification. Splenic homogenate and whole blood were serially diluted and plated on tryptic soy agar (TSA) plates. In other studies, mice were exposed to aerosolized Escherichia coli 0111:B4 LPS $(300 \mu \mathrm{g} / \mathrm{mL}$, Sigma-Aldrich) for 30 minutes, as previously reported (17). For airspace analysis, BALF was spun at $300 g$ for 6 minutes. Cells were then resuspended in PBS and counted. Cytospins were stained and differentials counted by light microscopy. In order to evaluate peritoneal trafficking of PMNs, mice were injected intraperitoneally with $0.5 \mu \mathrm{g}$ CXCL1, and euthanized 4 hours later for peritoneal lavage.

Generation of bone marrow chimeric mice. Procedures were followed as previously described (17). Emp $2^{+/+}$mice that were congenic for CD45 (stock no. 002014; Jackson Laboratory) were used. Recipients were lethally irradiated (900 rad) by a Model 431 irradiator using a 137Cs source (JL Shepherd and Associates). Within 4 hours after irradiation, donor-derived bone marrow from femurs and tibias $\left(2 \times 10^{6}\right.$ cells $)$ was injected i.v. into recipients. The efficiency of donor stem cell engraftment was determined by flow cytometry for CD45.1 $\left(E m p 2^{+/+}\right) 9$ weeks after transfer on circulating PMNs $\left(\mathrm{Gr}-1^{+}\right)$and B lymphocytes $\left(\mathrm{B} 22 \mathrm{O}^{+}\right)$. Engraftment efficiency within all experimental animals was greater than $90 \%$. Chimeras were used a minimum of 12 weeks after transplant.

Flow cytometry and fluorescence-activated cell sorting. Lung tissues were perfused, digested, and processed to single cell suspension as previously reported (56). Cells were blocked with anti-mouse CD16/32 (clone 2.4G2, Biolegend) and stained for 30 minutes with antibodies against: CD47 (clone miap301), ICAM-1 (clone 3E2), and integrin beta 3 (clone $\mathrm{HM} \beta 3-1$ ), all purchased from BD Biosciences. In some studies, cells were stained with $1 \mu \mathrm{g} / \mathrm{mL}$ cholera-toxin B-Alexa Fluor 488 (Thermo Fisher Scientific) to stain lipid rafts, as previously described (24). All flow cytometry analyses were performed on the LSR II (BD Biosciences) and analyzed using FACSDiva (BD Biosciences) and FlowJo software (Tree Star). For cell sorting, lungs were perfused, inflated, and digested with elastase (Worthington Biochemicals) for 45 minutes at $37^{\circ} \mathrm{C}$. Minced lung was then digested with DNase (MilliporeSigma) for 15 minutes. Cells were strained (70$\mu \mathrm{m}$ filter), depleted of CD $45^{+}$cells using AutoMACS column (Miltenyi Biotec), and then blocked with anti-mouse CD16/CD32 (clone 2.4G2) and normal mouse and rat serum (Jackson ImmunoResearch). Cells were incubated with one or more of the following antibodies/markers: CD31 (clone 390), CD34 (clone MEC14.7) from eBioscience/Thermo Fisher Scientific, and CD45 (clone 104), EpCAM/CD326 (clone G8.8), T1 $\alpha$ /podoplanin (clone 8.1.1), MHCII (clone AF6-120.1), as well as 7-AAD, all purchased from Biolegend. AT1 (CD45-CD31- CD34-EpC$\left.\mathrm{AM}^{\text {int }} \mathrm{T} 1 \alpha^{+} \mathrm{MHCII}^{-}\right)$, AT2 $\left(\mathrm{CD}^{-} 5^{-} \mathrm{CD} 31^{-} \mathrm{CD} 34^{-} \mathrm{EpCAM}^{\mathrm{int}} \mathrm{T}^{-} \alpha^{-} \mathrm{MHCII}^{+}\right)$, and airway epithelial cells $\left(\mathrm{CD} 45^{-} \mathrm{CD} 31^{-} \mathrm{CD} 34^{-} \mathrm{EpCAM}^{\mathrm{hi}} \mathrm{MHCII}^{-}\right)$, so identified as per a prior report (57), were then sorted on an ARIA-II flow cytometer (BD Biosciences) with purity greater than $92 \%-97 \%$. In some experiments, $\mathrm{CD}^{4} 5^{-} \mathrm{EpCAM}^{+}$cells were grown in tissue-culture plates for up to 9 days to induce AT1-like cells, as previously reported (58). Rat alveolar epithelial cells were purified from lung as previously reported (59) and cultured over multiple days.

Quantitation of pulmonary interstitial PMNs. A reported procedure was followed with minor modifications (60). In brief, LPS-exposed mice were injected i.v. with $10 \mu \mathrm{g}$ FITC-conjugated anti-CD45 (clone 30-F11) 5 minutes prior to sacrifice. Euthanized mice were exsanguinated via cardiac puncture, and then underwent BAL with approximately 2-3 mL $1 \times$ PBS and pulmonary vascular perfusion with approximately $10 \mathrm{~mL} 1 \times$ PBS. Lavaged and perfused lungs were excised and minced in the presence of $100 \mu \mathrm{g} / \mathrm{mL}$ unlabeled anti-CD45 (clone 30-F11) and digested in a cocktail of liberase/DNase/collagenase XI/hyaluronidase (60 minutes, $37^{\circ} \mathrm{C}$ ), followed by passage through a cell strainer. After erythrocyte lysis, cells were centrifuged ( $500 \mathrm{~g}, 5$ minutes), blocked ( $5 \%$ normal mouse serum, $5 \%$ normal rat serum, and $5 \mu \mathrm{g} / \mathrm{mL}$ [1:100] Fc blocker in FACS buffer), stained with APC-conjugated anti-Ly6G (clone 1A8), and resuspended for flow cytometry. Endovascular PMNs were identified as $\mathrm{CD}_{4} 5^{+} \mathrm{Ly}_{6 \mathrm{G}^{+}}$and interstitial (extravascular) PMNs as CD45-Ly6G ${ }^{+}$. In pilot studies, we confirmed that no intravitally labeled (CD45 $)$ PMNs were detectable in BALF.

Live lung slice imaging. Lungs ( $n=3-4$ mice/genotype) were harvested, sliced, stained (anti-CD31, -CD324/E-cadherin, and -Ly-6G), and imaged by confocal laser scanning microscope (Zeiss LSM 880), as previously described (61).

Immunofluorescence studies of fixed lung tissue. Lung tissue was fixed, embedded in paraffin, sectioned, and immunostained for GFP (Abcam ab13970), EMP2 (rabbit serum, a gift from Madhuri Wadehra [UCLA, Los Angeles, CA, USA]), SPC (Santa Cruz sc-7706), T1 $\alpha$ (University of Iowa Developmental Studies Hybridoma Bank 8.1.1), CCSP (62), and acetylated tubulin (MilliporeSigma, T7451), as previously described (63). Images were captured on a Leica SP5 confocal or a Zeiss Axioplan 2.0 microscope.

Quantitative RT-PCR. Total RNA was extracted from cells and tissues with RNeasy kits (Qiagen) using the manufacturer's protocol. For tissue-extracted RNA, tissues were homogenized with a TissueLyser (Qiagen). For sorted lung epithelial cells or cultured primary AT1-like cells, the RNAqueous-Micro Total RNA Isolation kit (Thermo Fisher Scientific) was used with elution in a $20 \mu \mathrm{L}$ volume. Generally, 1001000 ng RNA was converted to cDNA and used to perform real-time PCR (qPCR) reactions on Applied Biosystems ViiA 7 in duplicate. Predeveloped, validated primer/probe sets (Applied Biosystems) were used. The efficiency (slopes) of the target amplification and the efficiency of the reference endogenous control (GAPDH) amplification were $100 \%$ ( $\pm 10 \%)$.

Cell lines and generation of stable cells. Cell lines Calu-3 and NCI-H292 were purchased from ATCC, and Let1 cells were a gift from Paul Thomas at St. Jude Hospital, Memphis, TN, USA (26). CMT64 cells were purchased from MilliporeSigma. For stable knockdown of EMP2 in Calu-3 and NCI-H292, lentiviral-based pLKO vectors that carry scrambled or EMP2-targeted shRNA were purchased from Sigma-Aldrich to transduce both cell lines. Stable transduced cells were selected and maintained in medium containing $2 \mu \mathrm{g} / \mathrm{mL}$ puromycin (InvivoGen). Doxycycline-inducible Cav2 knockdown cells were generated in the EMP2 knockdown Calu-3 stable line by transduction of a lentiviral vector EZ-Tet-pLKO-Blast (Addgene, ref. 64) that express caveolin-2 shRNA targeting sequence (TRCNO000123022; TRCNO000296146). To express EMP2 WT or mutants in Let1 cells, EMP2 cDNA was pur- 
chased, and point mutations were introduced to CRAC or CARC sites in EMP2 DNA sequence and then cloned into lentiviral vector pLV-EF1aIRES-Puro (Addgene). Let1 cells were transduced by established lentiviral constructs with puromycin selection at a concentration of $2 \mu \mathrm{g} / \mathrm{mL}$.

In vitro studies of neutrophil transepithelial migration. Blood was collected from normal, healthy human donors. Procedures were followed as previously reported, with minor modifications (65). Briefly, Calu- 3 cells $\left(0.55 \times 10^{6} /\right.$ well $)(65)$ were seeded on the undersurface of polycarbonate membranes in Transwell tissue-culture inserts (Corning) and then grown to confluence (transepithelial resistance $\geq 1000$ $\Omega \cdot \mathrm{cm}^{2}$ ). Three million human neutrophils, freshly isolated by discontinuous plasma-Percoll gradient centrifugation (66), were added to the upper chamber, and $0.5 \mathrm{nM}$ fMLP (Sigma-Aldrich) was added to the lower chamber. Transmigration was allowed to proceed for 30-150 minutes, after which PMNs were quantified in the lower chamber with a quantitative myeloperoxidase assay (67).

Fluorescence microscopy. Cultured cells were stained with cholera toxin B and imaged by microscopy as previously reported (41).

Cytokine analysis. Cytokines were quantified by multiplex assay (Bio-Plex; Bio-Rad Laboratories) or ELISA (eBioscience).

$B A L F$ protein measures. The manufacturer's instructions (Pierce) were followed for the BCA protein assay, with colorimetric reading at OD562 on a Bio-Tek Synergy 2 microplate reader. Albumin and IgM were quantified by ELISA (Bethyl).

Immunoblotting. For detection of EMP2 protein, cell lysates were treated with PNGase (New England Biolabs; 60 minutes, $37^{\circ} \mathrm{C}$ ) as previously reported (14). Equal protein mass from cell lysates was run on a $10 \%$ sodium dodecyl sulfate-polyacrylamide gel and transferred to a PVDF membrane using standard methods. The membrane was probed with rabbit anti-EMP2 (1:1000; MilliporeSigma) and anti- $\beta$-actin-HRP (1:10000; MilliporeSigma) or mouse anti-Tubulin (1:1000; MilliporeSigma). Additional antibodies used at 1:1000 dilution include rabbit anti-Claudin-3, rabbit anti-Claudin-12, rabbit antiClaudin-18, rabbit anti-JAM-A, and mouse anti-Occludin, all from Invitrogen/ThermoFisher Scientific. Membranes were then washed and exposed (60 minutes) to 1:5000 HRP-conjugated secondary antibody (GE Healthcare) in 5\% milk/buffer. After further washes, signal was detected with ECL Western Blot detection reagents (GE Healthcare), followed by film exposure (GE Healthcare).

Histopathologic analysis. Tissues were fixed in 10\% neutral buffered formalin, trimmed, processed for paraffin, embedded, sectioned $(5 \mu \mathrm{m})$, and stained with H\&E. The slides were scanned using an Aperio slide scanner (Leica Biosystems) and images were captured using Aperio's ImageScope. Lung inflammation and injury were evaluated by a board-certified veterinary pathologist.

Statistics. Analysis was performed using GraphPad Prism software. Data are represented as mean \pm SEM. Two-tailed Student's $t$ test or Mann-Whitney test was applied for comparisons of 2 groups and 1-way ANOVA for comparisons of more than 2 groups. Survival was evaluated by log-rank test. For all tests, $P$ less than 0.05 was considered significant.

Study approval. All experiments were performed in accordance with the Animal Welfare Act and the U.S. Public Health Service Policy on Humane Care and Use of Laboratory Animals after review by the National Institute of Environmental Health Sciences (NIEHS) Animal Care and Use Committee. Collection of blood from normal, healthy donors was performed after obtaining written informed consent and was in accordance with an NIEHS institutional review boardapproved protocol.

\section{Author contributions}

KMG, WCL, JPM, and MBF designed, conducted, and analyzed experiments and contributed to the writing of the manuscript. RLZ performed the IF studies, assisted with the in vitro and transmigration studies, and contributed to writing. KY, JPM, and HN assisted in epithelial cell flow cytometry and sorting studies and contributed to writing. MLC and DNC conducted the live lung slice imaging studies and contributed to writing. $\mathrm{KJ}$ contributed to IHC, pathology evaluation, and writing. CJW contributed essential reagents and contributed to study design and writing. JHM performed and analyzed the in vivo pulmonary exposure experiments. KMG made the initial core findings and critical in vivo follow up investigations, whereas WCL played a larger role in later mechanistic studies and in manuscript completion; thus, WCL is listed first in the authorship.

\section{Acknowledgments}

The authors thank Ligon Perrow for assistance with breeding, Deborah King for blood cell count analysis, the NIEHS histology core laboratory for assistance with processing, sectioning, and staining of lungs, and Carl Bortner and Maria Sifre of the NIEHS Flow Cytometry Core Facility. We thank Madhuri Wadehra for providing anti-EMP2 antibodies. This research was supported by the Intramural Research Program of the NIH, National Institute of Environmental Health Sciences (Z01 ES102005).

Address correspondence to: Michael B. Fessler, National Institute of Environmental Health Sciences, 111 T.W. Alexander Drive, P.O. Box 12233, MD D2-01, Research Triangle Park, North Carolina 27709, USA. Phone: 984.287.4081; Email: fesslerm@niehs.nih.gov.

KMG's current address is: Department of Pharmacology and Toxicology, Brody School of Medicine, East Carolina University, Greenville, North Carolina, USA.
1. Rossaint J, Zarbock A. Tissue-specific neutrophil recruitment into the lung, liver, and kidney. J Innate Immun. 2013;5(4):348-357.

2. Burns AR, Smith CW, Walker DC. Unique structural features that influence neutrophil emigration into the lung. Physiol Rev. 2003;83(2):309-336.

3. Mizgerd JP. Molecular mechanisms of neutrophil recruitment elicited by bacteria in the lungs. Semin Immunol. 2002;14(2):123-132.

4. Zemans RL, Colgan SP, Downey GP. Transepi- thelial migration of neutrophils: mechanisms and implications for acute lung injury. Am J Respir Cell Mol Biol. 2009;40(5):519-535.

5. Li Q, Park PW, Wilson CL, Parks WC. Matrilysin shedding of syndecan-1 regulates chemokine mobilization and transepithelial efflux of neutrophils in acute lung injury. Cell. 2002;111(5):635-646.

6. Brazil JC, Parkos CA. Pathobiology of neutrophil-epithelial interactions. Immunol Rev. 2016;273(1):94-111.
7. Wadehra M, Iyer R, Goodglick L, Braun J. The tetraspan protein epithelial membrane protein-2 interacts with beta1 integrins and regulates adhesion. J Biol Chem. 2002;277(43):41094-41100.

8. Wadehra M, Su H, Gordon LK, Goodglick L, Braun J. The tetraspan protein EMP2 increases surface expression of class I major histocompatibility complex proteins and susceptibility to CTL-mediated cell death. Clin Immunol. 2003;107(2):129-136. 
9. Wadehra M, Goodglick L, Braun J. The tetraspan protein EMP2 modulates the surface expression of caveolins and glycosylphosphatidyl inositol-linked proteins. Mol Biol Cell. 2004;15(5):2073-2083.

10. Wadehra M, et al. Epithelial membrane protein-2 regulates surface expression of alphavbeta3 integrin in the endometrium. Dev Biol. 2005;287(2):336-345.

11. Fu M, et al. Epithelial membrane protein-2 promotes endometrial tumor formation through activation of FAK and Src. PLOS ONE. 2011;6(5):e19945.

12. Dahlin K, et al. Identification of genes differentially expressed in rat alveolar type I cells. Am J Respir Cell Mol Biol. 2004;31(3):309-316.

13. Fessler MB, Parks JS. Intracellular lipid flux and membrane microdomains as organizing principles in inflammatory cell signaling. J Immunol. 2011;187(4):1529-1535.

14. Williams CJ, et al. Epithelial membrane protein 2 (EMP2) deficiency alters placental angiogenesis, mimicking features of human placental insufficiency. J Pathol. 2017;242(2):246-259.

15. Shimazaki K, et al. Blockade of epithelial membrane protein 2 (EMP2) abrogates infection of Chlamydia muridarum murine genital infection model. FEMS Immunol Med Microbiol. 2009;55(2):240-249.

16. Yamamoto K, et al. Type I alveolar epithelial cells mount innate immune responses during pneumococcal pneumonia. J Immunol. 2012;189(5):2450-2459.

17. Draper DW, Madenspacher JH, Dixon D, King DH, Remaley AT, Fessler MB. ATP-binding cassette transporter $\mathrm{G} 1$ deficiency dysregulates host defense in the lung. Am J Respir Crit Care Med. 2010;182(3):404-412.

18. Rebres RA, Green JM, Reinhold MI, Ticchioni $\mathrm{M}$, Brown EJ. Membrane raft association of CD47 is necessary for actin polymerization and protein kinase $\mathrm{C}$ theta translocation in its synergistic activation of T cells. J Biol Chem. 2001;276(10):7672-7680.

19. Rai P, et al. Streptococcus pneumoniae secretes hydrogen peroxide leading to DNA damage and apoptosis in lung cells. Proc Natl Acad Sci U S A. 2015;112(26):E3421-E3430.

20. Moon C, Han JR, Park HJ, Hah JS, Kang JL. Synthetic RGDS peptide attenuates lipopolysaccharide-induced pulmonary inflammation by inhibiting integrin signaled MAP kinase pathways. Respir Res. 2009;10:18.

21. Humlicek AL, Pang L, Look DC. Modulation of airway inflammation and bacterial clearance by epithelial cell ICAM-1. Am J Physiol Lung Cell Mol Physiol. 2004;287(3):L598-L607.

22. Sumagin R, Robin AZ, Nusrat A, Parkos CA. Transmigrated neutrophils in the intestinal lumen engage ICAM- 1 to regulate the epithelial barrier and neutrophil recruitment. Mucosal Immunol. 2014;7(4):905-915.

23. Ridger VC, Wagner BE, Wallace WA, Hellewell PG. Differential effects of CD18, CD29, and CD49 integrin subunit inhibition on neutrophil migration in pulmonary inflammation. JImmunol. 2001;166(5):3484-3490.

24. Lai L, et al. MicroRNA-33 regulates the innate immune response via ATP binding cassette transporter-mediated remodeling of membrane microdomains. J Biol Chem. 2016;291(37):19651-19660.

25. Murphy AJ, et al. ApoE regulates hematopoietic stem cell proliferation, monocytosis, and monocyte accumulation in atherosclerotic lesions in mice. J Clin Invest. 2011;121(10):4138-4149.

26. Rosenberger CM, et al. Characterization of innate responses to influenza virus infection in a novel lung type I epithelial cell model. J Gen Virol. 2014;95(Pt 2):350-362.

27. Fantini J, Barrantes FJ. How cholesterol interacts with membrane proteins: an exploration of cholesterol-binding sites including CRAC, CARC, and tilted domains. Front Physiol. 2013;4:31.

28. Epand RM. Cholesterol and the interaction of proteins with membrane domains. Prog Lipid Res. 2006;45(4):279-294.

29. Sedzik J, Jastrzebski JP, Ikenaka K. Sequence motifs of myelin membrane proteins: towards the molecular basis of diseases. JNeurosci Res. 2013;91(4):479-493.

30. Fu C, He J, Li C, Shyy JY, Zhu Y. Cholesterol increases adhesion of monocytes to endothelium by moving adhesion molecules out of caveolae. Biochim Biophys Acta. 2010;1801(7):702-710.

31. Wan X, Chen Z, Choi WI, Gee HY, Hildebrandt F, Zhou W. Loss of epithelial membrane protein 2 aggravates podocyte injury via upregulation of caveolin-1. J Am Soc Nephrol. 2016;27(4):1066-1075.

32. Razani B, et al. Caveolin-2-deficient mice show evidence of severe pulmonary dysfunction without disruption of caveolae. Mol Cell Biol. 2002;22(7):2329-2344.

33. Lamaze C, Tardif N, Dewulf M, Vassilopoulos S, Blouin CM. The caveolae dress code: structure and signaling. Curr Opin Cell Biol. 2017;47:117-125.

34. Fra AM, Williamson E, Simons K, Parton RG. De novo formation of caveolae in lymphocytes by expression of VIP21-caveolin. Proc Natl Acad Sci U S A. 1995;92(19):8655-8659.

35. Le PU, Guay G, Altschuler Y, Nabi IR. Caveolin-1 is a negative regulator of caveolae-mediated endocytosis to the endoplasmic reticulum. J Biol Chem. 2002;277(5):3371-3379.

36. Han B, Copeland CA, Tiwari A, Kenworthy AK. Assembly and Turnover of Caveolae: What Do We Really Know? Front Cell Dev Biol. 2016;4:68.

37. Wang Y, et al. Hypoxia promotes ligand-independent EGF receptor signaling via hypoxia-inducible factor-mediated upregulation of caveolin-1. Proc Natl Acad Sci U S A. 2012;109(13):4892-4897.

38. Forbes A, et al. The tetraspan protein EMP2 regulates expression of caveolin-1. J Biol Chem. 2007;282(36):26542-26551.

39. Pereira PMR, et al. Caveolin-1 mediates cellular distribution of HER2 and affects trastuzumab binding and therapeutic efficacy. Nat Commun. 2018;9(1):5137.

40. Huber D, Balda MS, Matter K. Occludin modulates transepithelial migration of neutrophils. J Biol Chem. 2000;275(8):5773-5778.

41. Chowdhury SM, et al. Proteomic analysis of ABCA1-null macrophages reveals a role for stomatin-like protein-2 in raft composition and toll-like receptor signaling. Mol Cell Proteomics. 2015;14(7):1859-1870.
42. Hülsbusch N, Solis GP, Katanaev VL, Stuermer CA. Reggie-1/Flotillin-2 regulates integrin trafficking and focal adhesion turnover via Rab11a. Eur J Cell Biol. 2015;94(11):531-545.

43. Jin Y, Lee SJ, Minshall RD, Choi AM. Caveolin-1: a critical regulator of lung injury. Am J Physiol Lung Cell Mol Physiol. 2011;300(2):L151-L160.

44. Razani B, et al. Caveolin-1 null mice are viable but show evidence of hyperproliferative and vascular abnormalities. J Biol Chem. 2001;276(41):38121-38138

45. Drab M, et al. Loss of caveolae, vascular dysfunction, and pulmonary defects in caveolin-1 gene-disrupted mice. Science. 2001;293(5539):2449-2452.

46. Garrean S, et al. Caveolin-1 regulates NF-kappaB activation and lung inflammatory response to sepsis induced by lipopolysaccharide. J Immunol. 2006;177(7):4853-4860.

47. Murata T, et al. Reexpression of caveolin-1 in endothelium rescues the vascular, cardiac, and pulmonary defects in global caveolin-1 knockout mice. JExp Med. 2007;204(10):2373-2382.

48. Hu G, Ye RD, Dinauer MC, Malik AB, Minshall RD. Neutrophil caveolin-1 expression contributes to mechanism of lung inflammation and injury. Am J Physiol Lung Cell Mol Physiol. 2008;294(2):L178-L186.

49. Kumar D, et al. Genome-wide analysis of the host intracellular network that regulates survival of Mycobacterium tuberculosis. Cell.2010;140(5):731-743.

50. Kim HJ, Henke CA, Savik SK, Ingbar DH. Integrin mediation of alveolar epithelial cell migration on fibronectin and type I collagen. Am J Physiol. 1997;273(1 Pt 1):L134-L141.

51. Wilson HL, Wilson SA, Surprenant A, North RA. Epithelial membrane proteins induce membrane blebbing and interact with the $\mathrm{P} 2 \mathrm{X} 7$ receptor $\mathrm{C}$ terminus. JBiol Chem. 2002;277(37):34017-34023.

52. Barth K, Bläsche R, Neißer A, Bramke S, Frank JA, Kasper M. P2X7R-dependent regulation of glycogen synthase kinase $3 \beta$ and claudin- 18 in alveolar epithelial type I cells of mice lung. Histochem Cell Biol. 2016;146(6):757-768.

53. Root KT, Plucinsky SM, Glover KJ. Recent progress in the topology, structure, and oligomerization of caveolin: a building block of caveolae. Curr Top Membr. 2015;75:305-336.

54. Chung LK, et al. Epithelial membrane protein 2: Molecular interactions and clinical implications. JClin Neurosci. 2017;44:84-88.

55. Gee HY, et al. Mutations in EMP2 cause childhood-onset nephrotic syndrome. Am J Hum Genet. 2014;94(6):884-890.

56. Draper DW, et al. ATP binding cassette transporter G1 deletion induces IL-17-dependent dysregulation of pulmonary adaptive immunity. JImmunol. 2012;188(11):5327-5336.

57. Hasegawa K, et al. Fraction of MHCII and EpCAM expression characterizes distal lung epithelial cells for alveolar type 2 cell isolation. Respir Res. 2017;18(1):150.

58. Demaio L, et al. Characterization of mouse alveolar epithelial cell monolayers. Am J Physiol Lung Cell Mol Physiol. 2009;296(6):L1051-L1058.

59. Dobbs LG, Mason RJ. Pulmonary alveolar type II cells isolated from rats. Release of phosphatidylcholine in response to beta-adrenergic stimula- 
tion. JClin Invest. 1979;63(3):378-387.

60. Barletta KE, Cagnina RE, Wallace KL, Ramos SI, Mehrad B, Linden J. Leukocyte compartments in the mouse lung: distinguishing between marginated, interstitial, and alveolar cells in response to injury. JImmunol Methods. 2012;375(1-2):100-110.

61. Lyons-Cohen MR, Thomas SY, Cook DN, Nakano H. Precision-cut mouse lung slices to visualize live pulmonary dendritic cells. J Vis Exp. 2017;(122):55465.

62. Stripp BR, et al. Clara cell secretory protein deficiency alters clara cell secretory apparatus and the protein composition of airway lining fluid. Am J Respir Cell Mol Biol. 2002;27(2):170-178.

63. Jansing NL, McClendon J, Henson PM, Tuder RM, Hyde DM, Zemans RL. Unbiased quantitation of alveolar type ii to alveolar type i cell transdifferentiation during repair after lung injury in mice. $\mathrm{Am} J$ Respir Cell Mol Biol. 2017;57(5):519-526.

64. Frank SB, Schulz VV, Miranti CK. A streamlined method for the design and cloning of shRNAs into an optimized Dox-inducible lentiviral vector. BMC Biotechnol. 2017;17(1):24.

65. Zemans RL, et al. Neutrophil transmigration triggers repair of the lung epithelium via beta-catenin signaling. Proc Natl Acad Sci U S A. 2011;108(38):15990-15995.

66. Fessler MB, et al. Lipid rafts regulate lipopolysaccharide-induced activation of $\mathrm{Cdc} 42$ and inflammatory functions of the human neutrophil. J Biol Chem. 2004;279(38):39989-39998.

67. Parkos CA, Delp C, Arnaout MA, Madara JL. Neutrophil migration across a cultured intestinal epithelium. Dependence on a CD11b/CD18-mediated event and enhanced efficiency in physiological direction. JClin Invest. 1991;88(5):1605-1612. 